\title{
An Adaptive Medium Access Parameter Prediction Scheme for IEEE 802.11 Real-Time Applications
}

\author{
Estefanía Coronado, José Villalón, and Antonio Garrido \\ High-Performance Networks and Architectures (RAAP), Albacete Research Institute of Informatics (I3A), \\ University of Castilla-La Mancha, Albacete, Spain \\ Correspondence should be addressed to Estefanía Coronado; estefania.coronado@uclm.es
}

Received 28 July 2016; Revised 10 October 2016; Accepted 27 October 2016; Published 16 January 2017

Academic Editor: Francisco Martínez

Copyright (C) 2017 Estefanía Coronado et al. This is an open access article distributed under the Creative Commons Attribution License, which permits unrestricted use, distribution, and reproduction in any medium, provided the original work is properly cited.

\begin{abstract}
Multimedia communications have experienced an unprecedented growth due mainly to the increase in the content quality and the emergence of smart devices. The demand for these contents is tending towards wireless technologies. However, these transmissions are quite sensitive to network delays. Therefore, ensuring an optimum QoS level becomes of great importance. The IEEE 802.11e amendment was released to address the lack of QoS capabilities in the original IEEE 802.11 standard. Accordingly, the Enhanced Distributed Channel Access (EDCA) function was introduced, allowing it to differentiate traffic streams through a group of Medium Access Control (MAC) parameters. Although EDCA recommends a default configuration for these parameters, it has been proved that it is not optimum in many scenarios. In this work a dynamic prediction scheme for these parameters is presented. This approach ensures an appropriate traffic differentiation while maintaining compatibility with the stations without QoS support. As the APs are the only devices that use this algorithm, no changes are required to current network cards. The results show improvements in both voice and video transmissions, as well as in the QoS level of the network that the proposal achieves with regard to EDCA.
\end{abstract}

\section{Introduction}

Wireless technologies have experienced a marked increase in popularity over the past few years. As a result, the trend towards the use of wireless networks has been noticeable, and nowadays it is possible to find them in many different scenarios such as hospitals, airports, and universities. Due to their simplicity of deployment, low cost, and multimedia content support, IEEE 802.11 [1] networks have become essential and have reached a leading position in the market.

Access mode to the Internet and consumption patterns are also changing, especially those related to multimedia applications. In fact, the development of new video coding standards has led to an enhancement in the quality of the contents and a growth in the number of High Definition (HD) video streaming services. However, this improvement results in an increase in the volume of data that must be transmitted over the network. This new trend comes from the emergence of intelligent devices such as smartphones, tablets, and new generation game consoles. All these devices share a common feature, namely, being equipped with IEEE 802.11 interfaces.

Real-time applications have high Quality of Service (QoS) requirements, which are not provided by the original IEEE 802.11 standard. This drawback leads to developing the IEEE 802.11e amendment [2], where the QoS level is improved by introducing EDCA (Enhanced Distributed Channel Access) as a medium access function. EDCA is able to classify and prioritize the traffic by defining a group of medium access parameters. Nevertheless, some research proves that there are still some limitations in the QoS field that must be overcome, particularly with respect to voice and video transmissions.

In addition to stations with QoS support, IEEE 802.11e networks can be also composed of legacy stations. Legacy stations do not offer QoS capabilities and cannot make modifications to the medium access parameters. Accordingly, and with the aim of maintaining the interoperability between both types of stations, EDCA recommends the use of a group of values for these parameters. In spite of improving the 
performance of real-time applications, these values are not optimum for voice and video communications in a huge number of scenarios. For that reason, making an appropriate adjustment to these parameters becomes a key issue.

Artificial Intelligence (AI) techniques are developed to solve complex problems that usually require human reasoning. In this context, the use of such methods may be useful given the diverse conditions that can be found in a wireless network. In particular, the number of collisions of the network is one of the factors that determines the network status in a more significant way. Therefore, the application of AI techniques could make it possible to find traffic patterns and contribute to enhancing the QoS level and the performance of the network.

In this paper, we introduce a dynamic prediction scheme for the medium access parameters in EDCA to improve the QoS level over IEEE 802.11 WLANs. The suitable selection of the waiting time periods for every Access Category (AC) to access the channel leads to a reduction in the collisions in the network, mainly between voice and video frames. This strategy achieves, therefore, a double objective: on the one hand, the quality of the multimedia communications is considerably enhanced; on the other hand, it allows for a channel usage optimization. The major contribution of this paper is the capacity to adapt the EDCA configuration dynamically based on the traffic conditions by using a group of simple rules at the Access Point (AP). Thus, it only requires a few small modifications to the AP firmware, maintaining full compatibility with current commercial network cards.

The remainder of this paper is organized as follows. Section 2 reviews the IEEE 802.11e amendment and some proposals that aim to enhance the QoS level. Section 3 gives a summary of the Artificial Intelligence techniques used in this research. In Section 4 we introduce the proposed scheme and the most relevant design aspects, while in Section 5 the details of the implementation process are presented. Section 6 describes the final dynamic predictive model. As this proposal has been designed as an incremental process, the results of the performance evaluation and a comparison with EDCA are also discussed in this section. Finally, Section 7 provides some concluding remarks on our work.

\section{QoS in IEEE 802.11 Networks}

The original IEEE 802.11 standard introduces two functions to access the wireless medium, which cannot provide QoS capabilities. These functions are called Distributed Coordination Function (DCF) and Point Coordination Function (PCF). For this reason, the IEEE 802.11e amendment was developed.

2.1. IEEE 802.11e. The IEEE 802.11e amendment was released with the aim of providing QoS support to voice and video applications over IEEE 802.11 WLANs [2]. Actually, the main feature of this amendment is the capacity to differentiate traffic flows and services. As backward compatibility must be kept, a distinction is drawn between the stations that support QoS (QSTAs) and the stations that use DCF and do not offer such support (nQSTAs). For this purpose, the 802.11e amendment implements the Hybrid Coordination Function (HCF).
TABLE 1: Default EDCA parameter set for IEEE 802.11g PHY layer.

\begin{tabular}{|c|c|c|c|c|}
\hline $\mathrm{AC}$ & $\mathrm{CW}_{\min }$ & $\mathrm{CW}_{\max }$ & AIFSN & TXOP \\
\hline AC_BK & $\mathrm{aCW}_{\min }$ & $\mathrm{aCW}_{\max }$ & 7 & - \\
\hline AC_BE & $\mathrm{aCW}_{\min }$ & $\mathrm{aCW}_{\max }$ & 3 & - \\
\hline \multicolumn{2}{|c|}{ AC_VI $\left(\mathrm{aCW}_{\min }+1\right) / 2-1$} & $\mathrm{aCW}_{\min }$ & 2 & $3.008 \mathrm{~ms}$ \\
\hline \multicolumn{2}{|c|}{$\mathrm{AC}_{-} \mathrm{VO}\left(\mathrm{aCW}_{\min }+1\right) / 4-1$} & $\left(\mathrm{aCW}_{\min }+1\right) / 2-1$ & 2 & $1.504 \mathrm{~ms}$ \\
\hline
\end{tabular}

This function is composed of two channel access methods: HCF Controlled Channel Access (HCCA) and EDCA. As was the case with PCF, the first of them follows a centralized scheme to access the medium, while the second one works in a distributed way, as DCF does. To this end, the HCF implementation is mandatory for all the QSTAs. Nevertheless, only EDCA is supported by commercial network cards on current devices as a method for accessing the wireless medium.

EDCA improves the capabilities of DCF and distinguishes between eight User Priorities (UPs). Moreover, four ACs are defined, which are derived from the UPs and are in charge of classifying the traffic streams. In this way, in order to from highest to lowest priority, Voice (VO), Video (VI), Best Effort (BE), and Background (BK) Access Categories are considered, as sketched in Figure 1. Each AC works on its own transmission queue and is characterized by an EDCA parameter set. The EDCA parameter set specifies a priority level through an Arbitration Interframe Spacing Number (AIFSN) combination, a Transmission Opportunity interval (TXOP), and the duration of the Contention Window (CW). In order to provide a fair transmission for the DCF stations, the IEEE 802.11e amendment defines a standard combination of the medium access parameters, as shown in Table 1.

The Arbitration Interframe Spacing (AIFS) period determines the amount of time that a station must wait before beginning a new transmission. This is derived from the AIFS Number (AIFSN) value for each AC, as can be seen in (1) where the SlotTime denotes the duration of a slot according to the physical layer, and the Short Interframe Space (SIFS) refers to the amount of time used by high priority actions that require an immediate response.

$$
\text { AIFS }[\mathrm{AC}]=\text { AIFSN }[\mathrm{AC}] \cdot \text { SlotTime }+ \text { SIFS. }
$$

The CW size sets the length of idle time; after that a transmission of a given station may occur. The CW values are assigned in the inverse order to that of the priority of the corresponding AC. Whenever an unsuccessful transmission takes place, the CW follows an increment sequence in powers of two minus one. In this way, the size of CW could be increased until it reaches at most the value of $\mathrm{CW}_{\max }$. It must keep this value until a frame is successfully transmitted, CW being reset to $\mathrm{CW}_{\min }$. This algorithm is not exactly the same as that used by DCF stations [3]. In this case, whenever the medium is sensed busy after an AIFSN period, the counter previously mentioned is decreased by one time slot. Due to this change, the use of an AIFSN value of 3 time units by the $\mathrm{BE}$ traffic provides a similar priority to that offered to the DCF traffic. Likewise, TXOPs allows the transmission of multiple streams without gaining the medium access every time that a 


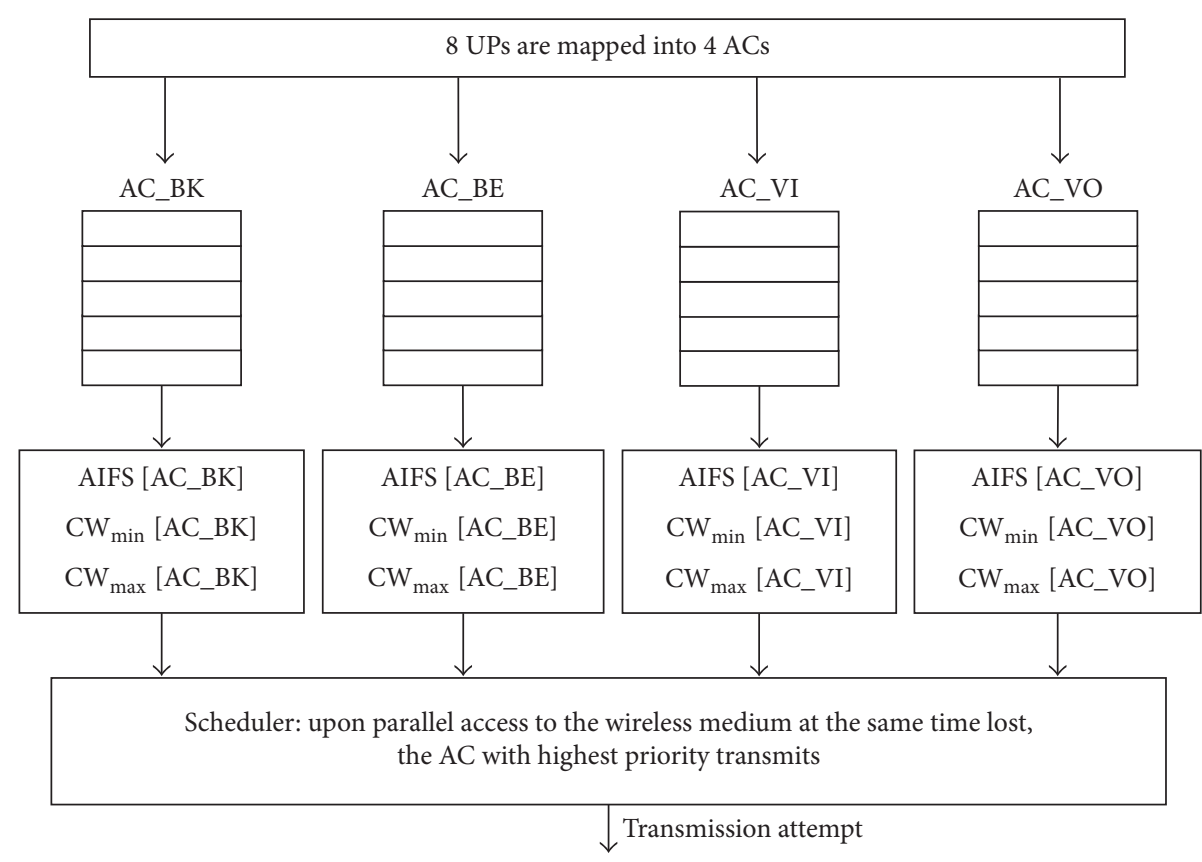

FIgURE 1: EDCA Access Categories mapping.

frame is transmitted. Therefore, they are usually used in realtime applications.

The AP of the network sends the EDCA parameter set through beacon frames to the stations of a Basic Service Set (BSS). The IEEE 802.11e amendment allows the APs to modify the values for this parameter set. However, no mechanism is considered in this amendment for carrying out this task and most commercial devices do not implement it.

2.2. Dynamic Adaptation in IEEE 802.11e. Wireless networks are conditioned by a huge set of factors that can change over time. For instance, the QoS level provided for all the services becomes worse as the congestion of the network and the number of collisions increase. In this regard, the usage of optimum values for the MAC parameters gains importance when trying to access the wireless medium in a more efficient way. In view of the above, several proposals have appeared with the aim of overcoming the QoS limitations that exist in IEEE 802.11 networks. They could be classified into three different categories: solutions designed at the link layer related to the medium access parameters, schemes designed at other layers such as the application level, and mathematical theories and formal models.

Recently, adapting the medium access parameters has taken increased importance. Depending on the selected values, the channel access can be more efficient, this being reflected in a significant reduction in the collisions and an increase in the QoS level. This aspect is of particular interest in the case of multimedia traffic transmitted in real-time due to their inherent temporal restrictions. This aspect has been studied in [4], in which it is shown the notable effect that using adequate priority parameters has on the network performance. After an analysis of these parameters using different priorities, it is shown that only the CW and the AIFS are associated with improvements, while the TXOP has no effect in the studied scenarios.

In this context, other authors have also examined this issue from a different point of view. In [5] a set of scenarios is presented in which several values for the AIFSN and CW are taken into account. Through this analysis, it is proved that the appropriate selection of the AIFSN combination contributes to reducing the collisions among the traffic of different ACs. Furthermore, the adequate tuning of the CW also leads to an enhancement of the QoS level. This conclusion is also reached in [6], where it is pointed out that a decrease in both the collisions and access media delay leads to an improvement in the efficiency of the network.

In [7] an adaptation scheme for the duration of CW is introduced, achieving better results than EDCA. However, compatibility with legacy DCF stations is not considered. To address this issue, another proposal presents a new way of offering backward compatibility with the DCF stations [8]. This algorithm prioritizes the voice and video traffic streams over the others. As the priority of the DCF stations cannot be modified by updating the EDCA parameter set, the CW size is increased by retransmitting packets that are properly received by the DCF stations. In this way, the priority of the stations that use EDCA decreases. Nevertheless, unnecessary traffic is introduced into the network. The tuning of CW is also taken into account in [9] for collision avoidance, outperforming the results of EDCA in terms of medium access delay.

An approach with this same goal is presented in [10], where three possible load levels are considered. This proposal achieves a reduction in the number of retransmissions and an enhancement in network performance. However, there is a drop in the voice and video traffic transmitted, which 
impairs its temporal restrictions. With the same aim, the approach proposed in [11] is based on the reservation and scheduling of multiple TXOPs, reducing in this way the number of collisions in the network. Nevertheless, using several consecutive TXOPs may penalize the remaining realtime applications, since they cannot access the channel for a certain amount of time.

Some other authors model their own medium access functions on the sidelines of EDCA or DCF. However, this type of models is usually incompatible with the operation mode of IEEE 802.11. In [12] a new function that combines the features of both DCF and EDCA functions is proposed. Indeed, it establishes a protocol that is able to determine the next transmitter according to a probability that depends on the priority of the traffic. Nevertheless, this approach cannot maintain interoperability with the current network cards.

Other approaches focus on ensuring an optimum level of QoS by carrying out any kind of optimization over other network layers. In [13] a middleware at the application level to improve the multimedia traffic performance is introduced. In particular, the main goal of this scheme is to maximize the provisioning quality to wireless clients in case of anomaly conditions in the network. Another scheme at this same level is proposed in [14]. iPAS is presented as a new way to adapt the priority of multimedia frames to establish an adequate bandwidth according to a group of QoS-related parameters. The architecture of this approach is composed of two main blocks: the iPAS server and the iPAS client. The iPAS client collects information about stream preferences, which is sent as feedback to the iPAS server. Then, based on these data, the iPAS server is responsible for managing bandwidth resources by means of a stereotype-based resource allocation mechanism and a bandwidth estimation scheme.

The design of an analytical model to improve network performance has also been considered. Nevertheless, most of these models make assumptions that may not be fulfilled in real transmissions. In [15] a model using Markov chains is defined. However, the same bit rate is considered for all the stations. By contrast, this issue is addressed by means of a bandwidth control scheme in [16]. In a similar way, the mathematical model presented in [17] is only tested under network saturation conditions. Finally, authors in [18] evaluate the model under an ideal channel scenario.

Although they are less common, besides Markovian models, $p$-persistent models have also been used. In [19] a time-domain analysis has been carried out in order to check the CSMA/CA performance and model the EDCA behavior from a different point of view. In spite of being properly validated via simulations, it is assumed that no transmission errors occur in the channel during the transmissions and all the stations always have a packet to send.

Although most of the described proposals outperform the results achieved by EDCA, it is not possible for all of them to maintain interoperability with legacy DCF stations. Actually, in many cases, these models experience compatibility problems with the IEEE 802.11 standard or introduce additional control traffic overhead in the network. In particular, despite this subject having been widely discussed, there are no other works that make use of Artificial Intelligence techniques to adapt the medium access parameters of EDCA.

\section{Supervised Learning}

Supervised learning refers to the task of defining a model, $h_{\Theta}(x)$, from supervised training data. The information relative to objects in this data is represented by a set of $n$ input features, $X=\left(X_{1}, \ldots, X_{n}\right)$, and an output variable, $Y$. In supervised learning, data is defined as a pair, $(X, Y)$, whose current outputs are already known (that is why it is called supervised). The process is in charge of analyzing the training data and using them to induce a model able to predict other unlabelled data $(y)$ when only the values of their input features $(x)$ are known.

The learning process may be of a different nature. In classification problems, the goal is to determine the class of an instance which is unknown $\left(Y \in\left\{c_{1}, \ldots, c_{K}\right\}\right)$. These models can be represented in several ways such as decision trees or classification rules. Regression analysis refers to a statistical methodology used in cases of numeric prediction. It is expected to identify distribution patterns in the current data, obtaining $Y \in \mathbb{R}$, and therefore $h_{\Theta}(x) \in \mathbb{R}$.

In this work, both types of supervised learning described above are utilized. $X$ represents the parameter configuration used for managing the voice and video traffic in a network, whereas the output $Y$ represents the throughput achieved by this setting. More specifically, in the case of the regression model, $h_{\Theta}(x) \in \mathbb{R}$ returns the predicted throughput of the network, $y$, given the parameter configuration $x$. By contrast, using the same configuration $x$, the classification model provides a label $Y$ for such an instance.

There are a large number of supervised learning models for regression, such as Linear Regression [20], Neural Networks [21], Support Vector Machines [22], or Regression Trees [23]. Selecting a certain model depends mainly on the aim of the application. Some of them are more powerful than others; in other words, they achieve greater precision and can detect more relevant patterns in data. However, the ease with which they can be interpreted can be an issue in some scenarios. Models such as Neural Networks are considered Black Box models due to the fact that the information related to underlying patterns in data cannot be extracted from them. In contrast, Regression Trees can be easily interpreted and provide useful information regarding the relation between input and output features. Another important issue concerns computational complexity. For instance, obtaining $y$ from $x$ with a Neural Network implies some matrix multiplications and can be too slow in some settings. However, processing a regression tree might only require a few comparisons. This becomes particularly important when using these models in real-time applications.

Due to the application domain of this work, it is important to select models that can quickly obtain the required result. The models must be used in real-time to determine the parameter setting that achieves a higher throughput. It is also important that the obtained models can be interpreted, analyzed, and even modified after having been learned. In the context of this work, a J48 classifier tree and a M5 regression 
model have been selected. They are further described in the sections below.

3.1. J48 Classifier Tree. The J48 classifier tree is based on the C4.5 algorithm, which is the successor to the ID3 algorithm [24]. This tree can be found in the weka package for machine learning [25]. This model aims to design a decision tree that is as short as possible. The algorithm follows a recursive procedure by means of a heuristic greedy search to obtain the final model. In this way, it selects every attribute according to its gain ratio (see (2)). This guideline expressly refers to the information gain obtained as a result of the classification made and the entropy of the predictive variable, $X_{i}$.

$$
\text { Gain ratio }=\frac{I\left(C, X_{i}\right)}{H\left(X_{i}\right)}=\frac{H(C)-H\left(C / X_{i}\right)}{H\left(X_{i}\right)} .
$$

The information gain is given by the expression $I\left(C, X_{i}\right)$, which obtains the mutual information between $X_{i}$ and $C$; that is, the algorithm evaluates the potential uncertainty when classifying an attribute $X_{i}$ on a set $C$. This is calculated as the difference between the entropy of the different outputs of the set $C, H(C)$, and the entropy obtained after using a certain attribute $X_{i}, H\left(C, X_{i}\right)$. In order to prevent the variables with a wider range of possible values from being the biggest beneficiaries in the classification, the information gain is weighted with the entropy of the predictive variable, $H\left(X_{i}\right)$.

The algorithm divides the training set into several subsets that are as pure as possible until a leaf node is reached. In this respect, the following internal node to be selected is the attribute which maximizes the aforementioned gain ratio. Once the tree has been modeled, this algorithm also incorporates a pruning technique to reduce its size and complexity. In the context of this work, an example of a subtree of the J48 classifier designed can be observed in Figure 2. In this way, it is possible to calculate the label for a certain combination which is situated at the leaf nodes. This label is obtained by using the rest of the parameters as an entry point for the tree.

3.2. M5Rules. The $M 5$ algorithm [23] represents $h_{\Theta}(x) \in \mathbb{R}$ as a regression tree and is very similar to its counterpart, $c 4.5$ [24], which is used for classification problems. This tree represents a division of the input space and each node defines a condition over some input attribute $X_{i}$. For instance, a node defined by the condition [VI_channel_occupancy $<=$ $0.341]$ represents the branch which would be used to process all objects whose value for variable VI_channel_occupancy is smaller than 0.341. Meanwhile, the other branch would be used to process the remaining cases. Each leaf represents an input subspace and corresponds to the cases which fit the conditions represented by the path from the root of the tree to the leaf.

In $M 5$, there are two possibilities to obtain the output values for the cases falling into a leaf of the tree. The first one, namely, regression tree, uses the mean output value of the training data falling into that leaf as default prediction. The second one, namely, model tree, learns a multivariate Linear Regression equation from the training data corresponding to the leaf and uses it to predict the output values.

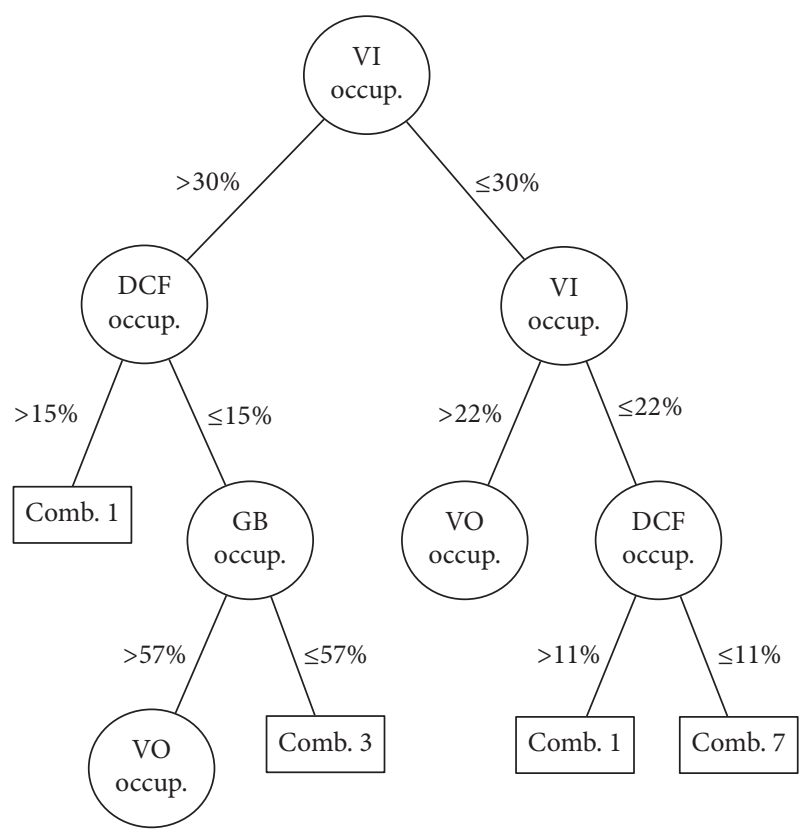

FIGURE 2: Example of subtree obtained by the J48 classifier tree.

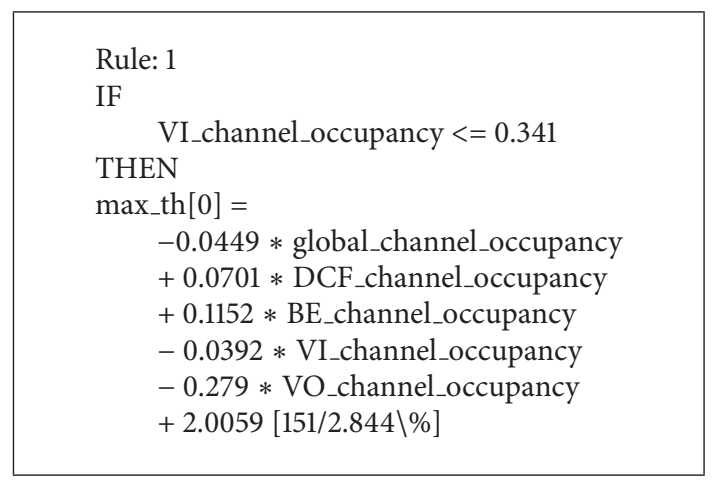

Algorithm 1: Example of rule induced by M5Rules.

The algorithm M5Rules is also included in the weka package. It first learns a regression (or model) tree from training data by means of the implementation of M5 included in this package, namely, M5P, and then extracts a set of rules. Algorithm 1 shows an example of the rules obtained. So that the value of all objects for variable [VI_channel_occupancy $<=$ $0.341]$; the output value is obtained as a linear expression from the remaining (5) variables. The information [151/ $2.844 \backslash \%$ ] indicates that 151 objects of the training dataset fall into that leaf and that the relative error obtained for those objects is $2.844 \%$.

\section{MAC Parameter Tuning Design}

In recent times, the number of applications with high QoS level requirements has increased as a result of the improvement in the quality of the multimedia contents. Consequently, traffic patterns in wireless networks have also changed significantly. 
Voice and video traffic transmitted in real-time is especially sensitive to latency and packet losses. Therefore, it must be given an even higher priority. The IEEE 802.11e amendment and the introduction of EDCA allow the definition of medium access parameters for each traffic type. However, due to the complexity involved in determining the traffic conditions at every moment, this amendment uses a default combination for these parameters instead of providing a mechanism for their adaptation. Moreover, the aforementioned parameters cannot be tuned in the case of legacy DCF stations. For this reason, compatibility with these stations becomes an important issue.

This paper presents a dynamic tuning scheme for the medium access parameters in EDCA to enhance QoS differentiation, targeted for real-time applications. The priority assigned to each AC is variable and depends on the traffic conditions. These priority levels are calculated by employing Artificial Intelligence techniques which consider both traffic flow patterns and network status. Furthermore, the scheme is fully compatible with EDCA, not being necessary to make changes to current network cards. Moreover, it seeks to ensure backward interoperability between the stations that use EDCA and those that use DCF.

4.1. Design Considerations. In order to optimize the performance of EDCA, it has been demonstrated that the AIFSN and the CW parameters are the most relevant factors [46]. Accordingly, the proposal focuses on determining the most suitable values for these parameters and adjusting them dynamically over time. In this way, it is possible to provide a more efficient mechanism to access the wireless medium. This improvement, in turn, allows it to reduce the collisions among the streams of both different and the same ACs. This leads to an enhancement in the QoS level, mainly for the voice and video traffic, due to its particular requirements for transmission in real-time.

Our scheme aims to improve the performance of realtime applications in Wi-Fi networks by reducing the number of collisions. One of the most serious difficulties in EDCA is precisely the huge amount of collisions that take place between voice and video transmissions, which are usually caused because they use the same AIFSN value and a very short length for the $\mathrm{CW}_{\min }$ and $\mathrm{CW}_{\max }$ parameters. In this context, a reduction of the number of collisions can be addressed in two ways: (i) increasing the separation between the AIFSN values for the voice and video applications and (ii) rising the length for the $\mathrm{CW}_{\min }$ and $\mathrm{CW}_{\max }$ parameters.

From the point of view of the AIFSN combination, the increase of the values must be performed on the video AC given that voice transmissions have stricter time constraints. The usage of AIFSN values higher than 2 slots for the video AC allows the voice applications to be provided with longer exclusive time periods to access the channel. Accordingly, collisions between voice and video frames would be reduced. Moreover, when voice packets collide and must be retransmitted, they could be long delayed or even discarded because of reaching the maximum deadline. As a consequence, the user experience would be highly damaged. On the other hand, if the AIFSN value for the video AC is increased, the values for the BE and BK ACs must be accordingly increased in an equal or greater proportion in order not to impair the video transmissions. However, if the network is partially composed of stations that use the original IEEE 802.11 standard (i.e., they use the DCF function), these legacy stations would have a higher priority to access the channel than the video applications, hence resulting in a noticeable decrease in the QoS performance.

Regarding the CW size, an increase of this parameter would directly lead to a reduction in the number of collisions in the network with respect to EDCA. This matter takes on particular importance for the voice and video traffic due to the short values defined for these ACs in EDCA. However, a significant enlargement of CW size would involve an increase in the waiting time to access the wireless medium. As a result, the real-time applications would be the most affected by this modification and the legacy stations would acquire even greater priority.

As can be seen, transmissions in wireless networks can be determined by several factors. For this reason, the complexity involved in determining the network conditions and tuning the medium access parameters according to them is considerably high. To perform this task, the most relevant factors are described below.

(i) Number of Active Applications of Each Type of Traffic. This parameter can be identified in a simple way by the AP. However, this is insufficient at a particular moment because it cannot provide further information about the current conditions of the network: that is, the scheme will not be allowed to obtain real information about the current occupancy of the wireless channel.

(ii) Application Bit Rate. Linked to the previous one, this factor provides more detailed information about the wireless medium status. Unfortunately, it is difficult to calculate in real-time. To identify these values it is necessary to introduce periodic control traffic in the network. Nevertheless, this feature is not typically used in IEEE 802.11e.

(iii) Transmission Rate. Each station may carry out its transmissions by using a different transmission rate. Therefore, the specific period of time that each of them keeps the channel busy is different. This parameter would be a good way of estimating the network conditions. Nonetheless, this value needs to be used jointly with the above factors.

(iv) Presence or Absence of Legacy DCF Stations. The existence of DCF applications restricts the use of priority parameters in EDCA, given that these values cannot be duly adjusted for these stations.

(v) Occupancy Level of the Wireless Medium. The amount of time that each traffic type keeps the medium busy allows us to obtain a good approximation of the network conditions. Indeed, the approximation that this only parameter can provide in a simple way is similar to that obtained by taking together all the previously described factors. 


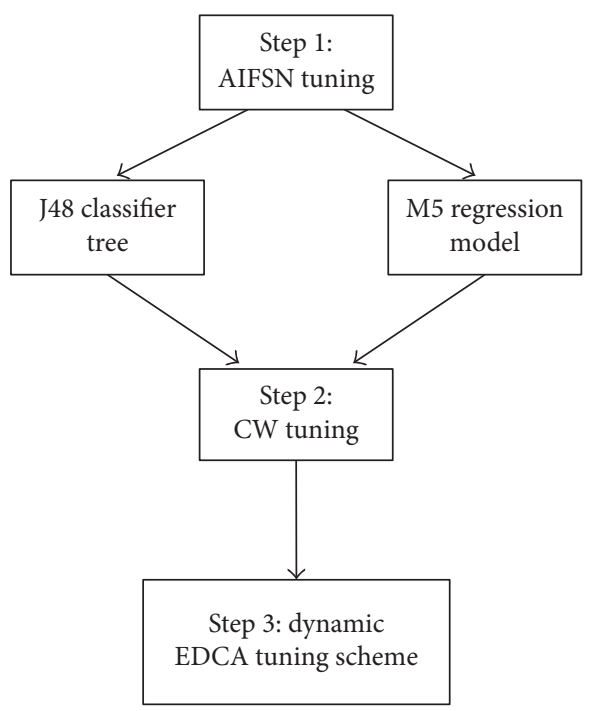

FIgURE 3: Dynamic EDCA prediction scheme's phases.

The proposed method requires to be used in real-time contexts without degrading the network performance, so the model should not be computationally complex. In this way, the medium access configuration can be recalculated at short intervals to achieve more accurate results. In this regard, AI techniques may be useful in identifying traffic patterns and determining optimum priority values for each AC. Furthermore, these techniques make it possible to simplify the huge amount of information that is involved in determining the network conditions. In this way, it is possible to handle only the information which plays a critical role in ascertaining the channel status.

4.2. Proposal Description. Given the presented constraints, our scheme takes into account the considerations described above. Firstly, the algorithm selects the optimum AIFSN combination depending on the current channel conditions. After that, and based on these values, the scheme calculates accordingly the most appropriate size for the CW parameter.

To ensure a better QoS differentiation, this proposal is divided into three phases, as depicted in Figure 3. The first phase involves the adaptation of the AIFSN combination through the design of two independent predictive models: an M5 regression model and a J48 classifier tree. To design these models, a deep training step must be performed. This training must consider a wide range of network situations to ensure that the models are built as close as possible to real environments. The prediction obtained from these models is used in the second phase, when the optimization of the CW size is carried out. In the third phase, all the designed models are unified into a single scheme which is able to discern the use of every one of them according to the network conditions. The appropriate selection of these priority values makes it possible to reduce both the internal and external collisions. This result is especially suitable for the multimedia traffic enhancement. Moreover, the usage of the wireless channel is optimized and, as a consequence, the network performance is also improved.

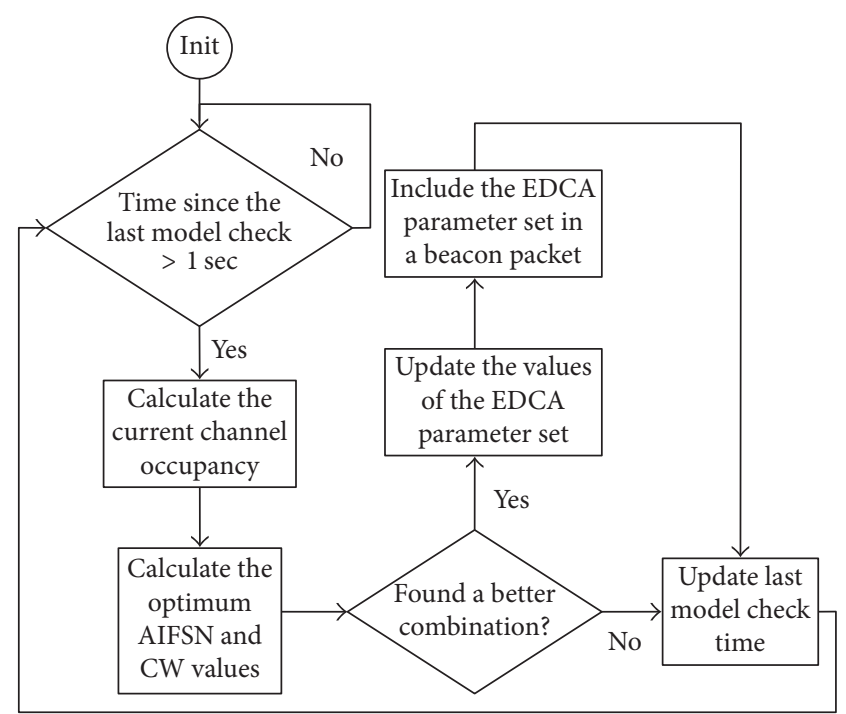

FIGURE 4: MAC parameters update process.

The proposed scheme is loaded in the APs of the network by introducing a few modifications in the firmware. In this regard, the predictive models, which are already trained and built, are ready to be used at any time. Therefore, it ensures the direct application on real environments, where the information about the network conditions is only used as input for the predictive scheme to obtain and update the appropriate EDCA values.

Traffic conditions may change rapidly, so the periodic update of the priority parameters becomes an important matter to consider. After evaluating different amounts of time, this recalculation period has been set to one second. Within the selected time, the AP of the network must determine the optimum priority values of that very moment and check whether they are equal to the current ones. If any difference is found, the AP must notify these new parameters to the stations. Once the optimum EDCA combination has been found, the updated information is embedded in an EDCA parameter set and transmitted via beacon frames. In this way, it avoids introducing additional control traffic into the network. The process described above can be observed in Figure 4.

The beacon interval is usually set to $100 \mathrm{~ms}$; therefore, if required, the values could be updated at every interval. However, channel conditions do not usually suffer relevant changes in such a short period and it would only cause abrupt modifications in the parameters that would not allow the algorithm to converge properly. The tests performed to set this period showed that the results were similar for updating periods from 1 to 5 seconds, these being less accurate when selecting a longer interval due to the excessive amount of time without updating the network information. Given the similarity among the previous periods, the final selection of a one-second interval was also due to the intention of implementing a scheme as appropriate for real environments as possible, where noises or movements may change suddenly the channel status. 
TABLE 2: Features of the dynamic adaptation schemes.

\begin{tabular}{|c|c|c|c|c|c|c|c|}
\hline & $\begin{array}{l}\text { IEEE } 802.11 \\
\text { compatibility }\end{array}$ & $\begin{array}{l}\text { Additional } \\
\text { traffic }\end{array}$ & Efficiency & Prioritization & $\begin{array}{c}\text { QoS } \\
\text { performance } \\
\text { with DCF and } \\
\text { EDCA stations }\end{array}$ & $\begin{array}{c}\text { Designed for } \\
\text { saturated traffic }\end{array}$ & Main issues \\
\hline Our proposal & Yes & No & Efficient & AIFSN + CW & $\begin{array}{l}\text { Higher than } \\
\text { EDCA }\end{array}$ & No & - \\
\hline i-EDCA [7] & Yes & No & Efficient & CW & $\begin{array}{l}\text { Lower than } \\
\text { EDCA }\end{array}$ & No & $\begin{array}{c}\text { Bad result in the } \\
\text { presence of DCF } \\
\text { stations. Only uses } \\
\text { CW }\end{array}$ \\
\hline DACKS [8] & Yes & No & Very inefficient & CW & $\begin{array}{l}\text { Higher than } \\
\text { EDCA }\end{array}$ & No & $\begin{array}{l}\text { Very inefficient. } \\
\text { Retransmits correct } \\
\text { packets }\end{array}$ \\
\hline AEDCA [9] & Yes & No & Efficient & CW & $\begin{array}{l}\text { Lower than } \\
\text { EDCA }\end{array}$ & No & $\begin{array}{c}\text { Bad results in the } \\
\text { presence of DCF } \\
\text { stations. Only uses } \\
\text { CW }\end{array}$ \\
\hline $\begin{array}{l}\text { MMDP- } \\
\text { FMAC } \\
{[10]}\end{array}$ & Yes & No & Efficient & Fair allocation & $\begin{array}{l}\text { Lower than } \\
\text { EDCA }\end{array}$ & No & $\begin{array}{l}\text { Bad results in the } \\
\text { presence of DCF } \\
\text { stations. Problems } \\
\text { with the deadline of } \\
\text { the real-time } \\
\text { applications }\end{array}$ \\
\hline $\begin{array}{l}\text { Hamidian and } \\
\text { Körner [11] }\end{array}$ & Yes & No & Efficient & TXOP & $\begin{array}{l}\text { Similar to } \\
\text { EDCA }\end{array}$ & No & $\begin{array}{l}\text { Problems with the } \\
\text { deadline of the } \\
\text { real-time applications }\end{array}$ \\
\hline QHDCF [12] & No & Yes & Efficient & $\begin{array}{l}\text { Centralized } \\
\text { priority }\end{array}$ & $\begin{array}{l}\text { Higher than } \\
\text { EDCA }\end{array}$ & No & $\begin{array}{c}\text { Incompatible with the } \\
\text { standard. Uses } \\
\text { modified packets }\end{array}$ \\
\hline iPAS [14] & No & Yes & Inefficient & $\begin{array}{l}\text { Centralized } \\
\text { priority }\end{array}$ & $\begin{array}{l}\text { Lower than } \\
\text { EDCA }\end{array}$ & No & $\begin{array}{l}\text { Incompatible with the } \\
\text { standard. Requires } \\
\text { stations changes. } \\
\text { Introduces additional } \\
\text { control traffic }\end{array}$ \\
\hline $\begin{array}{l}\text { Banchs and } \\
\text { Vollero [17] }\end{array}$ & Yes & No & Efficient & CW & $\begin{array}{l}\text { Lower than } \\
\text { EDCA }\end{array}$ & Yes & $\begin{array}{c}\text { Bad results in the } \\
\text { presence of DCF } \\
\text { stations. Only uses } \\
\text { CW. }\end{array}$ \\
\hline
\end{tabular}

Table 2 compares the main features of our scheme with those described in Section 2.2. The Table shows that some proposals have compatibility issues with the IEEE 802.11 standard. Therefore, these schemes cannot be used in realworld scenarios. On the one hand, there is a set of approaches that do not consider the large number of wireless cards whose features are still based on the original IEEE 802.11. These schemes (i-EDCA, AEDCA, MMDP-FMAC, iPAS, and Banchs et al.) achieve very poor results in the presence of stations that use DCF. In other words, the QoS performance achieved by these approaches is lower than the one offered by EDCA. This problem arises from the modification of the channel access function in order to improve the performance of the voice and video applications. For that reason, the usage of these schemes when the network is also composed of legacy DCF stations involves an increase in the priority of these last stations with regard to the video applications.
On the other hand, most of the evaluated schemes achieve good results in terms of efficiency since they do not require introducing additional traffic in the network. However, DACKS and iPAS schemes should be noted as exceptions. To reduce the priority of the legacy stations, in DACKS not all the frames that are successfully transmitted are acknowledged. Consequently, many properly received frames are retransmitted, hence introducing unnecessary traffic in the network and increasing the CW size. Meanwhile, iPAS uses a dedicated control communication link to send feedback information. As a result, besides the data link, an additional one is established between server and clients for the control traffic.

\section{Deployment Process}

The design of the dynamic predictive model requires the prior implementation of the two first phases of the deployment. 
TABLE 3: Set of AIFSN values analysed.

\begin{tabular}{ccccccccccc}
\hline & S0 & S1 & S2 & S3 & S4 & S5 & S6 & S7 & S8 & S9 \\
\hline BK & 7 & 8 & 9 & 8 & 9 & 12 & 10 & 12 & 14 & 14 \\
BE & 3 & 4 & 5 & 4 & 5 & 6 & 6 & 8 & 10 & 12 \\
VI & 2 & 2 & 2 & 3 & 3 & 3 & 4 & 5 & 6 & 7 \\
VO & 2 & 2 & 2 & 2 & 2 & 2 & 2 & 2 & 2 & 2 \\
\hline
\end{tabular}

As outlined in Section 4, these phases allow the independent adaptation of the AIFSN combination and the CW size for every AC in EDCA. This section presents the development performed in the first and second phases. The first phase also includes the description of the steps that have been followed in the training and learning phases to model the initial schemes. Given that the results are shared from one phase to another, the evaluation of these preliminary parts is also included.

5.1. AIFSN Adaptation. The models of the first phase aim to optimize the channel access by identifying the most suitable AIFSN combination. In this regard, a J48 decision tree classifier and an M5 regression model have been developed. The modeling of these schemes has been carried out independently in order to compare their capabilities and analyze how the different factors of a network determine their performance. Further details concerning this analysis, the development process and the evaluation performance, can be found in our previous works [26, 27].

According to the analyses carried out in $[5,6]$, a group of 10 AIFSN configurations has been selected with the goal of being an appropriate alternative to the one established in EDCA. These combinations, which can be observed in Table 3, have been chosen on the basis of the requirements previously introduced in Section 4.1. A gradual increase in the waiting time among each AC has been carried out for that selection with the aim of reducing the number of collisions and to enhance the QoS performance of the network. In particular, these combinations could be grouped into three different categories, as detailed below.

In the first category, 3 groups of combinations that consider an AIFSN value equal to 2 slots for both the AC_VO and the AC_VI can be found. The usage of these values does not allow reducing the collisions between voice and video frames. However, this intends to analyze the effect that increasing the AIFSN values for the $\mathrm{BE}$ and $\mathrm{BK}$ traffic flows has in reducing the aforementioned collisions. This group of combinations is advisable in the presence of a considerable amount of DCF traffic given that the legacy stations would not have a higher priority than the video applications to access the channel.

The second division includes 3 sets of combinations that establish an AIFSN equal to 2 slots for the AC_VO and equal to 3 slots for the AC_VI. These values will considerably reduce the collisions between voice and video applications due to the reserved time slot for the voice streams. Moreover, with the aim of studying their effect on the real-time applications performance, different AIFSN values have been assigned to the AC_BE and AC_BK. The values considered in this group are particularly suitable for a low DCF traffic load because the

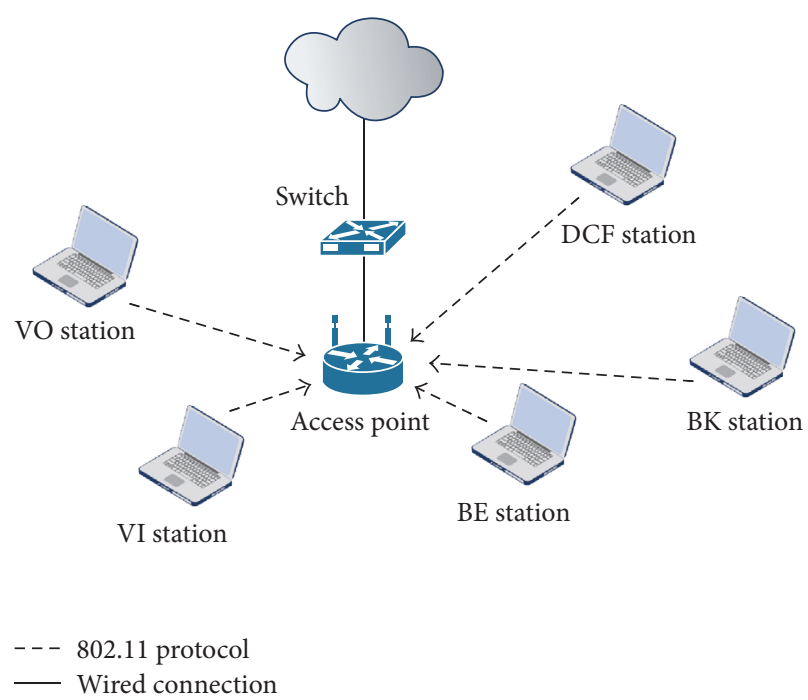

FIGURE 5: Network topology used in the deployment.

reduction achieved in the number of collisions compensates the decrease in the priority for the video transmissions.

The last group is composed of 4 sets of values that carry out a greater increase in the AIFSN value for the AC_VI than the previously performed. The usage of these combinations involves a greater reduction in the multimedia traffic collisions with respect to the previous ones. This is particularly beneficial for the voice applications given that they would have several time slots for their exclusive use. Nevertheless, it should be noted that these combinations are not recommended for the cases in which there is a huge number of legacy stations due to the reduction in the priority for the video transmissions.

The model design to predict the AIFSN combination starts with a training phase, which requires us to acquire a considerable amount of information. Accordingly, a huge set of tests covering a wide range of traffic conditions must be specified. This makes it possible to discover valuable knowledge and offer more precise predictions. In this work, the training tests are composed of 18 scenarios which have been modeled using Riverbed Modeler 18.0.0 [28]. In our simulations, we model an IEEE 802.11g wireless LAN cell comprising legacy DCF-based and EDCA stations. The EDCA stations support four different types of services: Voice (VO), Video (VI), Best Effort (BE), and Background (BK). The DCF-based stations support data traffic. We assume the use of a wireless LAN consisting of several wireless stations and an AP connected to a wired node that serves as sink for the flows from the wireless domain. All the stations are located within a Basic Service Set (BSS); that is, every station is able to detect the transmission from any other station. The parameters for the wired link were chosen to ensure that the bandwidth bottleneck of the system is within the wireless LAN (see Figure 5).

The scenarios are divided into two major groups: the first one considers both DCF and EDCA stations; whereas, the second group is only composed of stations that make use of EDCA. Accordingly, this proposal can ensure compatibility 
TABLE 4: Traffic proportions used for the training step.

\begin{tabular}{lccccc}
\hline \# scenario & VO & VI & BE & BK & DCF \\
\hline 1 & $20 \%$ & $20 \%$ & $20 \%$ & $20 \%$ & $20 \%$ \\
2 & $60 \%$ & $10 \%$ & $10 \%$ & $10 \%$ & $10 \%$ \\
3 & $30 \%$ & $30 \%$ & $10 \%$ & $10 \%$ & $20 \%$ \\
4 & $10 \%$ & $60 \%$ & $10 \%$ & $10 \%$ & $10 \%$ \\
5 & $30 \%$ & $20 \%$ & $10 \%$ & $10 \%$ & $30 \%$ \\
6 & $10 \%$ & $10 \%$ & $10 \%$ & $10 \%$ & $60 \%$ \\
7 & $10 \%$ & $10 \%$ & $20 \%$ & $20 \%$ & $40 \%$ \\
8 & $10 \%$ & $10 \%$ & $40 \%$ & $30 \%$ & $10 \%$ \\
9 & $50 \%$ & $50 \%$ & - & - & - \\
10 & $10 \%$ & $10 \%$ & $30 \%$ & $30 \%$ & $20 \%$ \\
11 & $60 \%$ & $40 \%$ & - & - & - \\
12 & $40 \%$ & $60 \%$ & - & - & - \\
13 & - & - & $40 \%$ & $30 \%$ & $30 \%$ \\
14 & $30 \%$ & - & $20 \%$ & $20 \%$ & $30 \%$ \\
15 & - & $30 \%$ & $20 \%$ & $20 \%$ & $30 \%$ \\
16 & - & - & $50 \%$ & $50 \%$ & - \\
17 & $20 \%$ & - & $40 \%$ & $40 \%$ & - \\
18 & - & $20 \%$ & $40 \%$ & $40 \%$ & - \\
\hline
\end{tabular}

with the legacy DCF stations. Each scenario starts with ten stations. In each scenario, the number of stations is increased from 10 to 80 in steps of 10 in order to increase the load of the wireless network. All the scenarios are composed of a variable percentage of applications of each type of traffic (BK, BE, VI, and VO). The traffic distributions used during the training phase can be seen in Table 4.

In order to adapt the medium access parameters in EDCA, the AP needs to know the channel occupancy rate at a given moment. This rate for the downlink traffic can be easily calculated by the AP. However, the main difficulty lies in estimating periodically the uplink traffic on the network. For this reason, the usage of only uplink traffic has been considered as a design decision. Nevertheless, the scheme can be also used in the presence of downlink traffic. In this case, both the uplink and the downlink occupancy rate are used as input for the algorithm to calculate the EDCA configuration.

In 802.11 WLANs stations use different rates, which determine the channel occupancy. Accordingly, the usage of a set of values such as 12 and $36 \mathrm{Mbps}$ has been considered for all the stations in the network, regardless of their AC. The remaining traffic features are unique to each type of traffic, as shown in Table 5. The BK, BE, and DCF traffic transmissions are modeled via a Pareto distribution with a location of 1.1 and a shape of 1.25 . The voice and video flows are represented by the transmission of G728 [29] and H.264 [30] streams, respectively. In the case of congestion, the network traffic is susceptible to experience delivery delays. Voice and video communications are more sensitive to the effects of this phenomenon than data transmissions. In this regard, deadline delays of 10 and $100 \mathrm{~ms}$ have been established for voice and video streams, respectively, beyond which these streams are discarded.

The simulations of the training phase are carried out by defining scenarios where the conditions of all the factors that
TABLE 5: Traffic parameters used for classifier construction.

\begin{tabular}{lcc}
\hline & Packet size & Data rate \\
\hline DCF & 552 bytes & $512 \mathrm{Kbps}$ \\
BK & 552 bytes & $512 \mathrm{Kbps}$ \\
$\mathrm{BE}$ & 552 bytes & $512 \mathrm{Kbps}$ \\
$\mathrm{VI}$ & 1064 bytes & $800 \mathrm{Kbps}$ \\
$\mathrm{VO}$ & 104 bytes & $20 \mathrm{Kbps}$ \\
\hline
\end{tabular}

determine them remain static. The values for such factors are modified according to a given order until all possible combinations have been considered. In this way, the predictive models are allowed to acquire real knowledge. In fact, if variable and random information were provided to the aforementioned models, the learning process would be unfeasible.

Although a considerable amount of information is needed to train the models, not all the parameters used are included. Some of them do not add any relevant information and merely made these models more complex. With the aim of designing accurate but simple classifiers, they must undergo a supervised variable selection process to discard those unrelated parameters. After carrying out this process, only the global occupancy level of the wireless channel and the particular level of each type of traffic are considered by the models.

Each predictive algorithm is provided a unique set of values. The J48 tree classifier only considers those AIFSN combinations that maximize the voice + video normalized throughput in each scenario. In contrast, the M5 regression model is given the information to all the AIFSN combinations, regardless of the throughput achieved. Since this model makes use of a group of regression functions to maximize the aforementioned throughput, this value must also be provided. As a result, the M5 model finally generates ten sets of submodels; that is, it contains one group of rules per AIFSN tested combination. This whole process makes it possible to obtain a good approximation of the network conditions while allowing the design of simple and accurate predictive models.

Both the preprocessing step and the design of the models have been carried out using Weka 3.7.0 [25]. As a part of the design process, a 10 -fold cross validation is performed to guarantee that both the training and the testing data sets are independent. This process results in a hit rate of $94.90 \%$ for the J48 tree. Meanwhile, the M5 model achieves an average correlation coefficient of 0.8916 and a mean absolute error of 0.0554 . These values show the accuracy of the proposed models and the strong relation among the parameters involved.

The second phase of the work and the tuning of the CW size take as a starting point the enhancements achieved by the first one in order to address the existing weaknesses. For this reason, a performance analysis of the models is required. This process is performed via simulation using Riverbed Modeler 18.0.0, through which 20 scenarios different from those used during the training step have been designed. The network topology used in the evaluation is the same as that presented in the training phase (see Figure 5). The scenarios are composed of 100 stations which use DCF and EDCA as medium 
TABLE 6: Description of the set of test scenarios.

\begin{tabular}{lccccc}
\hline \# scenario & Voice & Video & BE & BK & DCF \\
\hline 1 & $20 \%$ & $1.5 \%$ & $2 \%$ & $2 \%$ & $2 \%$ \\
2 & $20 \%$ & $5 \%$ & $2 \%$ & $2 \%$ & $2 \%$ \\
3 & $8 \%$ & $7 \%$ & $2 \%$ & $2 \%$ & $2 \%$ \\
4 & $16 \%$ & $6 \%$ & $3 \%$ & $3 \%$ & $7 \%$ \\
5 & $6 \%$ & $2 \%$ & $3 \%$ & $3 \%$ & $10 \%$ \\
6 & $6 \%$ & $3 \%$ & $4 \%$ & $4 \%$ & $8 \%$ \\
7 & $5 \%$ & $3 \%$ & $7 \%$ & $7 \%$ & $4 \%$ \\
8 & $5 \%$ & $6 \%$ & $10 \%$ & $5 \%$ & $5 \%$ \\
9 & $5 \%$ & $9 \%$ & $6 \%$ & $6 \%$ & $6 \%$ \\
10 & $8 \%$ & - & $8 \%$ & $8 \%$ & $8 \%$ \\
11 & - & $6 \%$ & $6 \%$ & $6 \%$ & $9 \%$ \\
12 & $5 \%$ & $6 \%$ & $6 \%$ & $6 \%$ & $6 \%$ \\
13 & $20 \%$ & $8 \%$ & - & - & - \\
14 & $9 \%$ & $4 \%$ & - & - & - \\
15 & $5 \%$ & $10 \%$ & - & - & - \\
16 & $6 \%$ & $7 \%$ & $7 \%$ & $7 \%$ & - \\
17 & $8 \%$ & - & $8 \%$ & $8 \%$ & - \\
18 & - & $8 \%$ & $7 \%$ & $7 \%$ & - \\
19 & $8 \%$ & $8 \%$ & $6 \%$ & $6 \%$ & - \\
20 & $8 \%$ & $7 \%$ & $8 \%$ & - & - \\
\hline
\end{tabular}

access functions. The first twelve scenarios consider both types of stations, while in the remaining eight only stations that support EDCA can be found. Furthermore, an equal proportion of stations of each type of traffic has been considered; that is, 20 stations per type of traffic have been included. However, not all the applications are active at the same time. On the contrary, they are enabled or disabled according to a probability that depends on their AC (see Table 6).

Every scenario takes 600 seconds to be simulated, with this simulation divided into two periods. During the first one, and every 30 seconds, the stations which are not transmitting any information try to start a new transmission according to the aforementioned probability. During the second period, the stations attempt to stop the current transmissions every 30 seconds, making use of the same probability. Due to all scenarios being simulated by using 60 random seeds and each of them being divided into 20 time intervals, in the end 24000 different intervals have been tested.

The traffic features for each station depend on the type of traffic it transmits. Regarding this, the bit rate and packet size used to verify the models are the same as those shown in Table 5. The stations are randomly distributed over the network coverage. Furthermore, the Ricean [31] model determines the signal propagation through the wireless medium. This model is characterized by a factor, $k$, which defines the ratio between the power in the line-of-sight component and the power in the scattered paths. In this work, a $k$ factor of 32 has been used. Moreover, IEEE 802.11g [32] defines the physical layer of the network. The stations use all the transmission rates defined in this amendment regardless of the traffic type.

The results allow it to compare the performance achieved by the proposed schemes and EDCA. To summarize these
TABLE 7: Voice + video normalized throughput improvements in $30 \mathrm{~s}$ intervals (AIFSN tuning).

\begin{tabular}{lcccc}
\hline & \multicolumn{2}{c}{ With DCF traffic } & \multicolumn{2}{c}{ Without DCF traffic } \\
& J48 & M5 & J48 & M5 \\
\hline Unaltered & $49.42 \%$ & $52.11 \%$ & $35.52 \%$ & $31.30 \%$ \\
Losses & $4.49 \%$ & $5.48 \%$ & $2.55 \%$ & $1.44 \%$ \\
Gain [1\%-5\%] & $27.20 \%$ & $23.37 \%$ & $23.64 \%$ & $17.35 \%$ \\
Gain [5\%-10\%] & $8.67 \%$ & $12.78 \%$ & $6.68 \%$ & $5.93 \%$ \\
Gain [10\%-15\%] & $6.06 \%$ & $2.86 \%$ & $7.41 \%$ & $6.89 \%$ \\
Gain [15\%-20\%] & $2.01 \%$ & $1.52 \%$ & $4.48 \%$ & $4.55 \%$ \\
Gain [from 20\%] & $2.16 \%$ & $1.90 \%$ & $19.73 \%$ & $32.55 \%$ \\
\hline
\end{tabular}

results properly, a group of statistics has been defined. These statistics show the voice + video normalized throughput, the number of retransmission attempts, the normalized throughput of the DCF applications, and the global throughput of the network. The first metric refers to the sum of the normalized throughput of both voice and video applications.

Table 7 shows the percentage of the 24000 time intervals evaluated in which the proposed models experience losses or gains with regard to EDCA. Notice that, especially if both DCF and EDCA applications are considered, almost half of the intervals remain unaltered. We have considered unaltered results to be those in which the gains or the losses are lower than $1 \%$. This situation is a consequence of the stations attempting to both start and finish their transmissions during the first and the last five simulation intervals. The traffic load in many of these cases is low, so all the AIFSN combinations achieve the highest throughput. Moreover, in the scenarios in which many stations use DCF, the default EDCA combination is the most efficient option. Therefore, in these scenarios the predictive schemes also use these values to access the medium.

Table 7 also presents some losses with regard to EDCA. Losses have been defined as those intervals in which the models experience a performance decrease higher than $1 \%$. These losses result from some certain cases in which our schemes miss the prediction. The use of a small number of parameters to design the models provides a good approximation of the network conditions. However, due to its simplicity, this image is not perfect, having as a consequence some errors in the parameter prediction. The aforementioned losses are also because the selection of a different AIFSN combination makes some minimum changes in the traffic status that might be considered as losses when they really are not. Nevertheless, the cases in which this phenomenon occurs are much fewer than those in which the schemes outperform EDCA.

Nevertheless, Table 7 shows how our models improve the performance achieved by EDCA in many more scenarios. These improvements are from $20 \%$ in some cases, being even higher if only EDCA applications are considered. Thus, in $19.73 \%$ and $32.55 \%$ of the intervals, enhancements of $20 \%$ are achieved when using the J48 and M5 models, respectively. Given that in some intervals the traffic load level does not allow the observation of the performance difference between the proposal and EDCA, Figure 6 presents the voice + video 


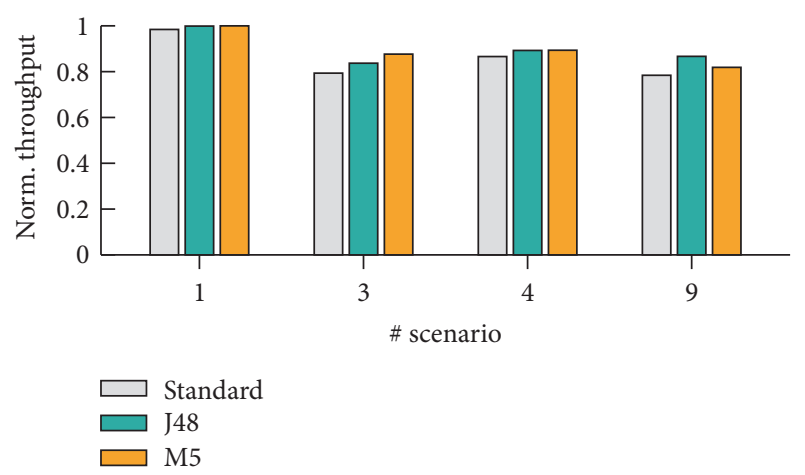

(a) Scenarios with EDCA and DCF stations

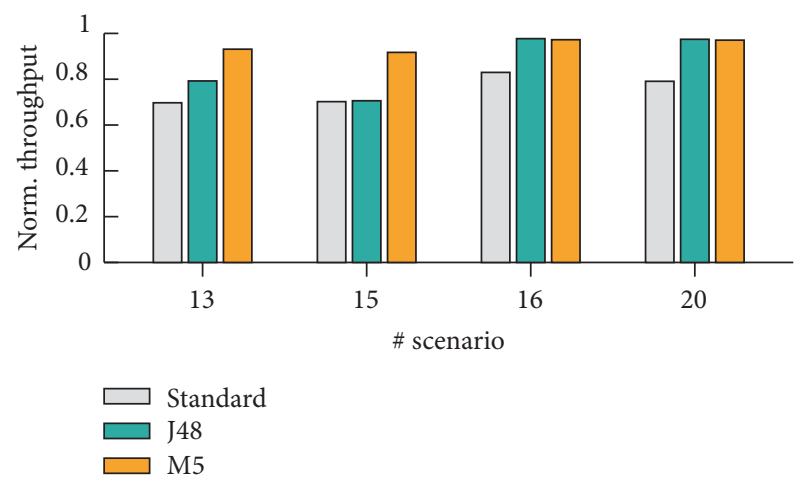

(b) Scenarios with EDCA stations

FIGURE 6: Voice + video norm. Throughput (AIFSN adaptation).

normalized throughput of the ten intervals in which traffic congestion becomes a key factor. In this figure, it is proved that in all the scenarios the predictive schemes outperform EDCA.

The proximity of the EDCA values, especially between the voice and video traffic, constrains the network performance due to the huge number of collisions. The appropriate AIFSN tuning of this approach allows it to optimize the channel access, resulting in a decrease in the number of collisions and retransmissions (see Figure 7). This is the main reason why the QoS level provided to real-time applications is enhanced. Furthermore, this improvement also leads to an increase in the overall throughput of the network, as can be seen in Figure 8.

A suitable separation of the AIFSN values, particularly in those cases in which the AIFSN for video traffic is higher than 2 slots, provides the stations that do not support QoS features with a higher priority to access the wireless medium. While ensuring compatibility with this type of stations, the proposed models improve their performance, as can be observed in Figure 9. It is important to point out that, despite this increase in the priority, the performance achieved by the multimedia communications is not only penalized but also enhanced in all the cases tested, as depicted in Figure 6.

Although both the presented predictive models enhance the performance of EDCA, they have certain features which make them quite different. On the one hand, the features of the J48 classifier allow it to achieve better results in the

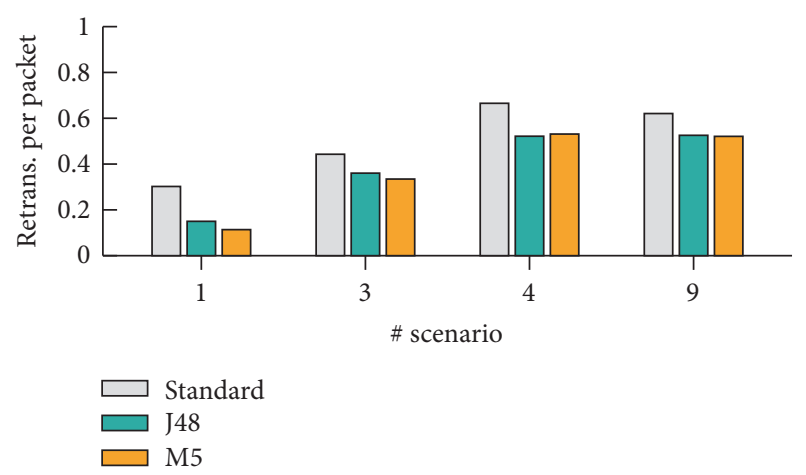

(a) Scenarios with EDCA and DCF stations

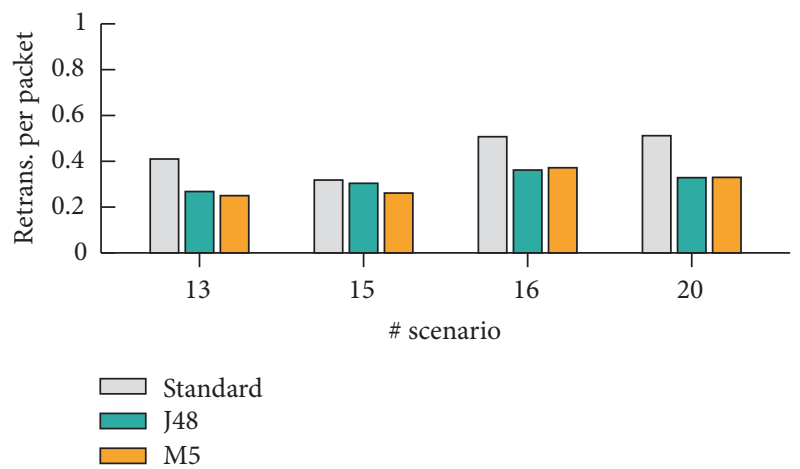

(b) Scenarios with EDCA stations

FIGURE 7: Overall retransmission attempts (AIFSN adaptation).

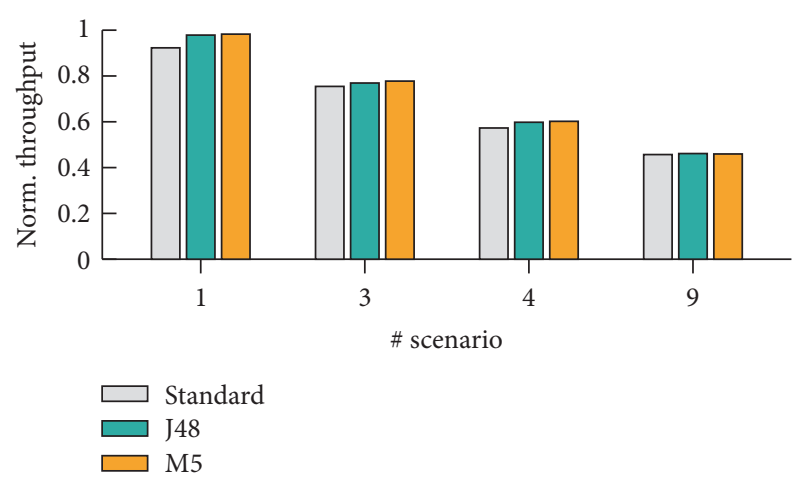

(a) Scenarios with EDCA and DCF stations

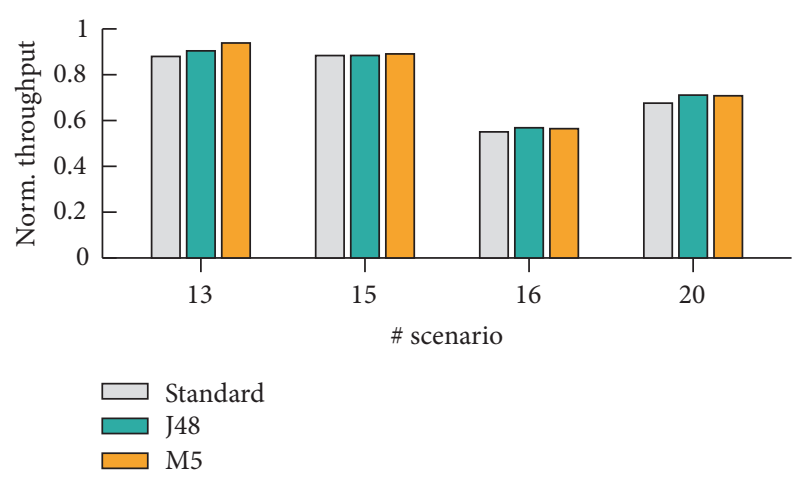

(b) Scenarios with EDCA stations

FIGURE 8: Global norm. Throughput (AIFSN adaptation). 
TABLE 8: Proposed values for the $\mathrm{CW}_{\min }$ and $\mathrm{CW}_{\max }$ limits.

\begin{tabular}{|c|c|c|c|c|}
\hline & \multicolumn{2}{|c|}{ CW 1} & \multicolumn{2}{|c|}{ CW 2} \\
\hline & $\mathrm{CW}_{\min }$ & $\mathrm{CW}_{\max }$ & $\mathrm{CW}_{\min }$ & $\mathrm{CW}_{\max }$ \\
\hline $\mathrm{BK}$ & $2 \cdot \mathrm{aCW}_{\min }+1$ & $\mathrm{aCW}_{\max }$ & $2 \cdot \mathrm{aCW}_{\min }+1$ & $\mathrm{aCW}_{\max }$ \\
\hline $\mathrm{BE}$ & $2 \cdot \mathrm{aCW}_{\min }+1$ & $\mathrm{aCW}_{\max }$ & $2 \cdot \mathrm{aCW}_{\min }+1$ & $\mathrm{aCW}_{\max }$ \\
\hline VI & $\mathrm{aCW}_{\min }$ & $2 \cdot \mathrm{aCW}_{\min }+1$ & $2 \cdot \mathrm{aCW}_{\min }+1$ & $2 \cdot \mathrm{aCW}_{\min }+1$ \\
\hline $\mathrm{VO}$ & $\left(\mathrm{aCW}_{\min }+1\right) / 2-1$ & $\mathrm{aCW}_{\min }$ & $\mathrm{aCW}_{\min }$ & $\mathrm{aCW}_{\min }$ \\
\hline
\end{tabular}

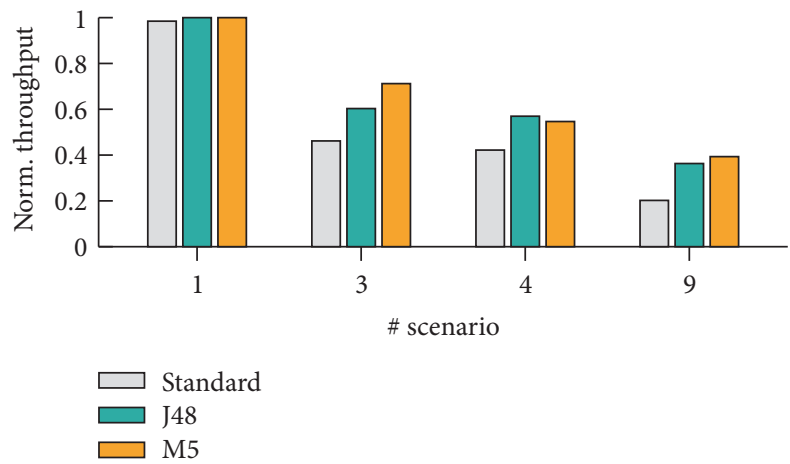

FIGURE 9: DCF norm. Throughput (AIFSN adaptation).

presence of a considerable load of DCF traffic. On the other hand, the gains experienced by the M5 model are higher in those scenarios in which DCF traffic is not considered or the number of these transmissions is relatively low. Moreover, in spite of the fact that neither model is computationally complex, the M5 regression model requires evaluating the voice + video normalized throughput for every AIFSN combination. Therefore, the J48 decision tree has a lower computational cost due to the minimum set of comparisons needed. This feature is especially important with regard to the execution of the medium access function in real-time.

5.2. CW Adaptation. The main aim of the second phase of this proposal is to further improve the multimedia communications through the reduction of the collisions among the flows of the same AC. Based on the evaluation performed in the first phase, it has been shown that in several scenarios there is still room for improvement. In this regard, in the second phase, the collision reduction problem is addressed by estimating the optimum values for the CW size without neither increasing severely the waiting time to access the channel nor reducing the priority of the real-time applications.

As is the case with the AIFSN, the IEEE 802.11e amendment establishes a set of values for the CW size (see Table 1). These values seek to ensure an appropriate QoS level, while maintaining compatibility with the legacy stations. Indeed, they aim to achieve good network performance when it hosts a considerable amount of legacy applications. In these cases, the selection of longer periods for the CW size would result in an earlier access to the medium by the DCF stations. In this way, the priority of this type of traffic would be increased with regard to video traffic, instead of being similar to that of the BE streams. For this reason, the adaptation of the CW parameter is of particular interest in scenarios where there is a low or null DCF traffic load.

Taking into account the huge number of collisions in $\mathrm{Wi}-$ Fi networks, mainly between voice and video transmissions due to the very small values of the $\mathrm{CW}_{\min }$ and $\mathrm{CW}_{\max }$ parameters, we have considered relevant to study the effect of carrying out these last increases. In order not to prejudice the priority of the multimedia applications the size of the $\mathrm{CW}_{\min }$ for AC_BE and AC_BK has been also enlarged. The $\mathrm{CW}_{\max }$ of these ACs has not been increased due to its high value.

Based on the prediction of the schemes designed in the first phase of the proposal, the $\mathrm{CW}_{\min }$ and $\mathrm{CW}_{\max }$ limits are increased with regard to the default EDCA values, as can be seen in Table 8. In the Backoff algorithm the CW size follows an incremental sequence of $2^{c}-1$ whenever a station needs to retransmit a frame. This factor is given special attention in this second phase, with the proposal of two approaches called $C W 1$ and $C W 2$.

The first modification of the $\mathrm{CW}$ size, called $C W 1$, increases the $c$ parameter by one with respect to EDCA for every AC. The only exception is the $\mathrm{CW}_{\max }$ limits for the $\mathrm{BE}$ and BK ACs, which maintain the default values. Given that these values are large enough, they are not modified with the aim of not introducing unnecessary waiting times into the network. The second version, called $C W 2$, follows a similar pattern to its predecessor. As the objective of this second proposal is to avoid the collisions between the voice and video traffic, only the duration of $\mathrm{CW}_{\text {min }}$ of these $\mathrm{ACs}$ is modified by increasing the $c$ parameter by two units. Furthermore, the $\mathrm{CW}_{\max }$ limits for all the ACs keep the same values as those used in the CW 1 approach. Notice that neither of the proposed configurations considers a reduction in the size of CW. This reduction, especially for the ACs whose limits are relatively small such as voice and video ACs, would result in a huge increase in the number of collisions between the frames of the same type of traffic.

An increase in the size of CW involves longer periods of time for the stations to finish the Backoff algorithm. More specifically, some combinations of AIFSN values and CW sizes may result in a considerable decrease in the priority of the stations that use EDCA with regard to those that use DCF. For this reason, the four approaches presented in Table 9 reduce the distance among the AIFSN values of all the ACs in order not to accumulate excessive waiting periods. All of these approaches aim to show the performance achieved when combining an increase in the length selected for the $\mathrm{CW}_{\min }$ and $\mathrm{CW}_{\max }$ parameters with the use of lower values from those predicted for the AIFSN combination. In this 
TABLE 9: Optimization configurations for the CW size and AIFSN combination.

\begin{tabular}{lcccc}
\hline & Configuration 1 & Configuration 2 & Configuration 3 & Configuration 4 \\
\hline AIFSN & Predicted & Predicted & Prev. combination & 2nd Prev. combination \\
CW & Default & CW 1 & CW 1 & CW 2 \\
\hline
\end{tabular}

way, the objective is to verify whether this strategy is able to outperform the results already achieved for voice and video communications. Finally, a total of eight predictive schemes are obtained given that each of the four proposed configurations can be used with both the J48 and the M5 models.

The first configuration proposed in Table 9, called Configuration 1, refers to the already modeled schemes in the first phase. In the remaining configurations, the adaptation of the CW size is carried out according to the values shown in Table 8. In this regard, Configuration 2 and Configuration 3 take the CW size of the combination $C W 1$. By contrast, Configuration 4 takes the values related to the $C W 2$ approach. Furthermore, the AIFSN combination can be chosen from among 3 different sets of values. Firstly, the prediction made for this parameter in the first phase can be maintained, as is the case with Configuration 1 and Configuration 2. Moreover, as is done for Configuration 3, another option is to select the AIFSN combination immediately below the one predicted by the J48 and M5 models. Likewise, the AIFSN combination can be tuned using the values provided by the second combination immediately below the predicted one. More specifically, this combination, which is used by Configuration 4, establishes a smaller spacing among the values of all the ACs. The selection of the aforementioned AIFSN combinations is carried out whenever possible, provided that they do not exceed the default EDCA one.

The real aim of this proposal is to design a dynamic prediction scheme for the EDCA parameter set. For this reason, in addition to the results obtained from the AIFSN tuning in the first phase, it is necessary to evaluate the performance achieved by these eight new preliminary schemes. In fact, the results of both the two phases will become the basis for developing the dynamic predictive model in the third phase. In order to carry out this task, we have considered the same simulation conditions and scenarios defined in the previous evaluation. In this way, it is possible to ensure a fair comparison of the results of both phases.

In Figure 10 the results in terms of voice + video normalized throughput are presented. The quality of the multimedia applications is mainly conditioned by the presence of stations that use DCF. This is due to the fact that a too large increase in the size of CW, particularly when the traffic load of the legacy stations is relatively high, has as a consequence a reduction in the performance of the voice and video transmissions. Despite these aspects, a small increment in the size of CW under low DCF traffic loads contributes to outperforming the results achieved by the J48 and M5 initial models. Nevertheless, this situation differs in scenarios where there are no stations which use the DCF function. In most of these cases, the usage of a larger size for CW makes it possible to improve the performance of the real-time applications. Various examples of this phenomenon can be observed in Figure 10.
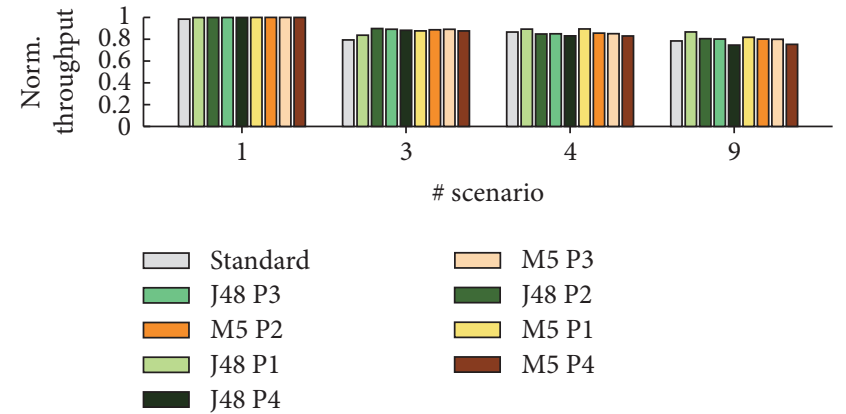

(a) Scenarios with EDCA and DCF stations

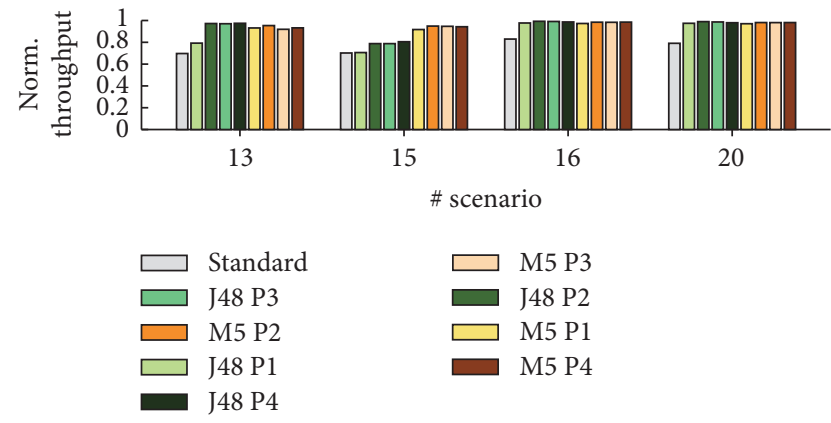

(b) Scenarios with EDCA stations

FIgURE 10: Voice + video norm. Throughput (CW adaptation).

The enhancements achieved in this second phase are mainly due to the reduction of both internal and external collisions among the traffic flows of the different ACs. As a consequence, a decrease in the number of retransmissions in the network is achieved. In the presence of a high number of stations that utilize the DCF function, using an AIFSN combination below the one that has been already predicted in the first phase of the proposal might result in an increase in the number of retransmissions. In spite of this fact, and as can be observed in Tables 10 and 11, the adequate selection of the size of CW allows the schemes to keep this number of retransmissions constant or even lower.

The increase in the waiting time to access the wireless medium for the video traffic flows has as a consequence an improvement in the performance of the legacy applications that use DCF. This phenomenon can be observed in Table 10, in which the legacy DCF stations achieve a higher throughput than in the first phase of the development. Nevertheless, this is not the real aim of these predictive schemes. In scenarios in which the DCF traffic load becomes high and the proposed approaches increase the duration of the CW parameter, the performance of these applications is improved, even if it is a secondary objective of the proposal. As a result, the global performance of the network is also enhanced. As it is shown 
TABLE 10: Overview of the CW adaptation results (scenarios with DCF and EDCA stations).

\begin{tabular}{lccccccccc}
\hline & Standard & J48 P1 & J48 P2 & J48 P3 & J48 P4 & M5 P1 & M5 P2 & M5 P3 & M5 P4 \\
\hline VO + VI Norm. Th. & 0.9259 & 0.9440 & 0.9225 & 0.9227 & 0.9016 & 0.9438 & 0.9312 & 0.9242 & 0.9022 \\
Retrans. per Pkt & 0.5447 & 0.4459 & 0.3469 & 0.3636 & 0.3662 & 0.4271 & 0.3473 & 0.3659 & 0.3724 \\
Global Norm. Th. & 0.6014 & 0.6199 & 0.6420 & 0.6395 & 0.6425 & 0.6225 & 0.6399 & 0.6379 & 0.6390 \\
DCF Norm. Th. & 0.5498 & 0.6790 & 0.7965 & 0.7743 & 0.7715 & 0.7118 & 0.8056 & 0.7876 & 0.7812 \\
\hline
\end{tabular}

TABLE 11: Overview of the CW adaptation results (scenarios with EDCA stations).

\begin{tabular}{lccccccccc}
\hline & Standard & J48 P1 & J48 P2 & J48 P3 & J48 P4 & M5 P1 & M5 P2 & M5 P3 & M5 P4 \\
\hline VO + VI Norm. Th. & 0.7759 & 0.8790 & 0.9415 & 0.9385 & 0.9419 & 0.9338 & 0.9596 & 0.9531 & 0.9549 \\
Retrans. per Pkt & 0.4604 & 0.3428 & 0.2271 & 0.2456 & 0.2234 & 0.3329 & 0.2288 & 0.2450 & 0.2267 \\
Global Norm. Th. & 0.6776 & 0.6965 & 0.7329 & 0.7320 & 0.7450 & 0.7010 & 0.7304 & 0.7296 & 0.7408 \\
\hline
\end{tabular}

in Table 11, the predictive schemes achieve an enhancement in terms of voice + video normalized throughput in the absence of legacy DCF stations, which in turn involves an improvement in the global performance of the network.

Following the evaluation performed in this second phase, it can be concluded that the adaptation of the size of CW keeps enhancing the quality of the multimedia transmissions. Nevertheless, it must be taken into account that an excessive increase in this parameter in the presence of a considerable number of stations that use DCF would penalize the performance of the video applications. In fact, in view of these situations, this increment should not be carried out.

Finally, the wide variety of results that have been obtained in both the already presented phases is clearly noticeable. As a consequence, it requires carrying out a deep analysis process to define in a precise way the network conditions in which the different approaches achieve the optimum performance. In fact, this analysis becomes the starting point for the development of the dynamic prediction scheme for the medium access parameters in EDCA.

\section{Dynamic EDCA Prediction Scheme}

In the previous section, four medium access parameter configurations are proposed for the J48 and M5 initial models (see Table 9). As a result, a total of 8 predictive schemes have been obtained. These schemes are named after the predictive model (M5 or J48) and the configuration used, for example, M5 Configuration 1 for the scheme that uses the first parameter configuration of the M5 model.

Given the wide variety of schemes and their achieved results, it is essential to unify them into a single one which is able to discriminate among all of them. This unification must be carried out through an exhaustive analysis of the results obtained. In this regard, a set of traffic patterns, which determines the structure of the dynamic prediction scheme has been identified. This structure is shown in Algorithm 2, where the behavior of the algorithm is mainly conditioned by the presence of DCF traffic. In spite of having designed 8 predictive models, not all of them are able to predict the medium access parameters accurately. Moreover, it has been proved

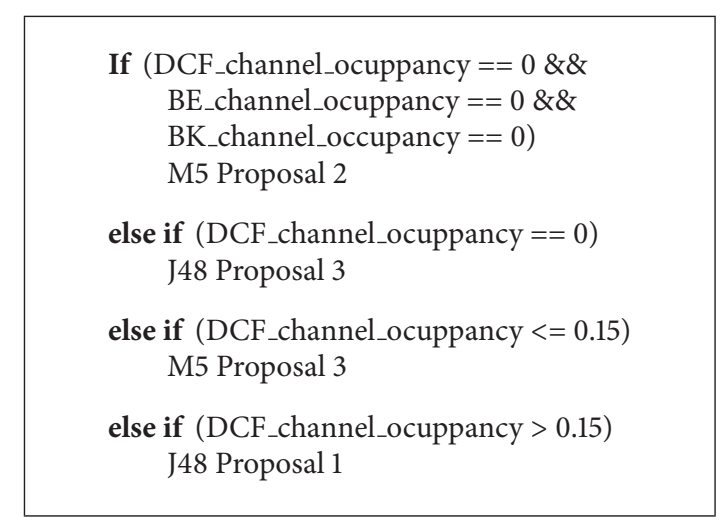

Algorithm 2: Structure of the dynamic prediction scheme.

that several schemes achieve similar results. Therefore, the final dynamic scheme is only composed of 4 submodels.

The analysis of the results consists of studying the parameter configurations that achieve the highest performance in each one of the proposed scenarios. After that, the results have been filtered in order to choose only the most suitable combination in every case. However, in some scenarios, several configurations provide similar results in accordance with the established traffic patterns. On this basis, and to simplify the model, only one of these potential candidates has been finally selected for the corresponding traffic pattern.

The evaluation highlights that, in the presence of DCF traffic, its occupancy level plays the most important role. If this level is higher than $15 \%$, the best results are provided by the first version of the J48 classifier (J48 Proposal 1). This model does not increase the size of CW, which is a proper decision that does not penalize the video applications when accessing the medium. In fact, in the first development phase, it was proved that, when the network carries a high DCF traffic load, the J48 model achieves better performance than the M5 one. By contrast, if this occupancy level is lower than 15\%, M5 Proposal 3 achieves the best performance. As this traffic level is relatively low, this selection is appropriate due to the fact that the increase in the $\mathrm{CW}$ size makes it possible to reduce the collisions among the frames of the same AC. 
TABLE 12: Voice + video normalized throughput improvements in $30 \mathrm{~s}$ intervals (dynamic scheme).

\begin{tabular}{lcc}
\hline & With DCF traffic & Without DCF traffic \\
\hline Unaltered & $56.37 \%$ & $34.27 \%$ \\
Losses & $2.46 \%$ & $1.19 \%$ \\
Gain [1\%-5\%] & $23.29 \%$ & $15.01 \%$ \\
Gain [5\%-10\%] & $6.49 \%$ & $5.95 \%$ \\
Gain [10\%-15\%] & $2.96 \%$ & $7.54 \%$ \\
Gain [15\%-20\%] & $2.94 \%$ & $6.19 \%$ \\
Gain [from 20\%] & $5.49 \%$ & $29.85 \%$ \\
\hline
\end{tabular}

By contrast, if there are no applications without QoS support, voice and video applications become the most important factor. When the network only carries voice and video traffic, M5 Proposal 2 provides the most accurate prediction. Given that this model keeps the AIFSN prediction and increases the $\mathrm{CW}$ size, it enables a reduction in the number of collisions among the multimedia streams and, as a result, an improvement in the performance of the network. Meanwhile, if other types of traffic are considered, the best option is to select the third version of the J48 classifier (J48 Proposal 3). This model offers a trade-off between the CW size and the differentiation given by the AIFSN values chosen. In this context, with the exception of the first proposal of this model which does not take into account the size of $\mathrm{CW}$, the remaining ones achieve a similar performance.

In addition to implementing the design presented above, the real adaptation capacity of the proposal must be verified. In this respect, to compare the performance achieved, the statistics show the results for EDCA, both the J48 and M5 initial models and the schemes of the second phase that achieve a higher voice + video normalized throughput. These latter schemes are denoted as $\mathrm{J} 48^{*}$ and $\mathrm{M} 5^{*}$. The results for the modeled dynamic scheme are also included.

Table 12 presents the results of the voice and video performance in terms of the percentage of time intervals into which simulations are divided. Notice that the percentage of unaltered intervals presents only minor changes with regard to previous evaluations (see Table 7). However, taking advantage of the strengths of each model makes it possible to reduce the amount of losses with regard to EDCA. In fact, the percentage of intervals in which any type of improvement is achieved reaches $41.17 \%$ and $64.54 \%$ in the presence and absence of DCF traffic, respectively. These enhancements are more meaningful when there are no stations without QoS support in the network, such enhancements being from $20 \%$ in $29.85 \%$ of the cases. In Figure 11 it can be seen that the dynamic model outperforms the remaining ones in almost all the tested scenarios. This results from properly choosing the prediction scheme that selects the most suitable parameter configuration at every moment.

The appropriate selection of the AIFSN combination and the CW size reduces the collisions in the network, which results not only in an increase in the voice + video normalized throughput but also in an improvement in the overall network performance (see Tables 13 and 14). The results indicate that

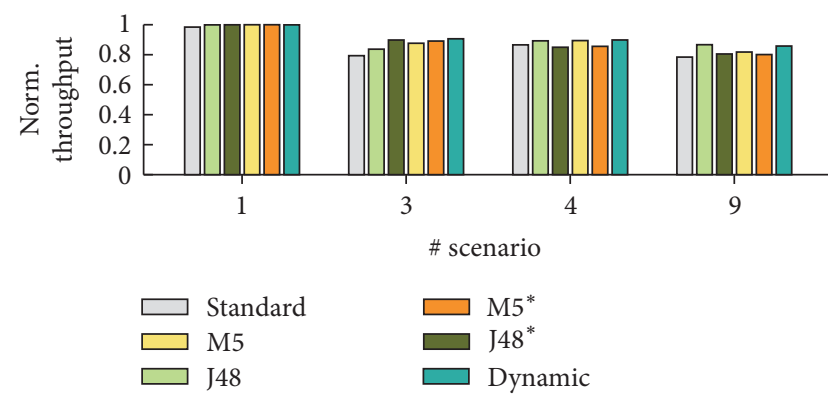

(a) Scenarios with EDCA and DCF stations

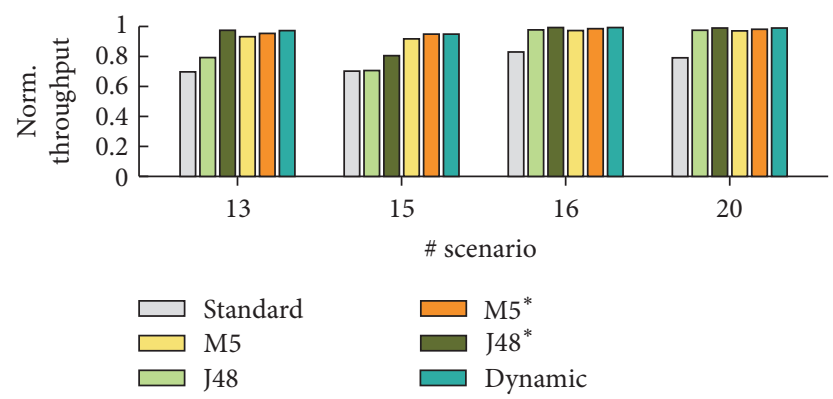

(b) Scenarios with EDCA stations

FIGURE 11: Voice + video norm. Throughput (dynamic adaptation).

the dynamic scheme reduces the number of collisions with regard to both EDCA and the models of the first phase (J48 and M5). However, this scheme obtains a greater number of collisions than the $\mathrm{J} 48^{*}$ and $\mathrm{M} 5^{*}$ approaches. This is due to the fact they penalize the real-time applications. The same reasoning applies to the enhancement of the global performance of the network.

The DCF traffic throughput has also been analyzed. As can be seen in Table 13, this throughput depends on its occupancy level in the network. If this value remains low, the performance of these stations is the same or better when using the dynamic model than when using EDCA. Nevertheless, if the network carries a considerable amount of DCF transmissions, the dynamic parameter combination allows it to transmit less DCF traffic than the remaining analyzed options. In particular, these last options damage heavily the performance of video applications. Therefore, the dynamic approach is logical, given that it seeks to improve the quality of the realtime transmissions.

To conclude this analysis, the improvement in terms of voice + video throughput achieved by our scheme is evaluated. The improvement shown is based on the traffic load of the voice and video applications. To that end, both the voice and video traffic flows have been divided into two groups: the first group contains the results for the 10 scenarios that hold the lowest voice traffic load (called low video traffic load), while the second one, referred to as high voice traffic load, is made up of the 10 scenarios with the highest voice traffic load. The same description applies to the video applications. During the performance analysis it has been proved that the existence of stations that use the DCF function plays a decisive role in the network performance. Therefore, the results are 
TABLE 13: Overview of the dynamic adaptation results (scenarios with DCF and EDCA stations).

\begin{tabular}{lcccccr}
\hline & Standard & J48 & J48* & M5 & M5* & Dynamic \\
\hline VO + VI Norm. Th. & 0.9259 & 0.9440 & 0.9227 & 0.9438 & 0.9312 & 0.9512 \\
Retrans. per Pkt & 0.5447 & 0.4459 & 0.3636 & 0.4271 & 0.3473 & 0.4267 \\
Global Norm. Th. & 0.6014 & 0.6199 & 0.6395 & 0.6225 & 0.6399 & 0.6263 \\
DCF Norm. Th. & 0.5498 & 0.6790 & 0.7743 & 0.7118 & 0.8056 & 0.7496 \\
\hline
\end{tabular}

TABLE 14: Overview of the dynamic adaptation results (scenarios with EDCA stations).

\begin{tabular}{lcccccr}
\hline & Standard & J48 & J48* & M5 & M5* & Dynamic \\
\hline VO + VI Norm. Th. & 0.9259 & 0.9440 & 0.9419 & 0.9438 & 0.9596 & 0.9683 \\
Retrans. per Pkt & 0.4604 & 0.3428 & 0.2234 & 0.3329 & 0.2288 & 0.2247 \\
Global Norm. Th. & 0.6776 & 0.6965 & 0.7050 & 0.7010 & 0.7304 & 0.7331 \\
\hline
\end{tabular}

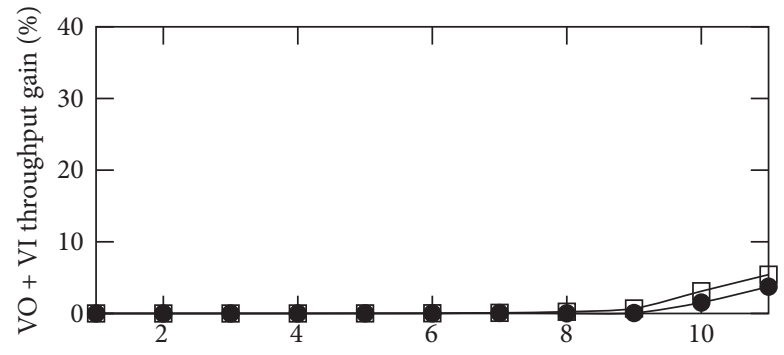

Network load (Mbps)

- Low voice traffic load

$\square$ High voice traffic load

(a) Scenarios with EDCA and DCF stations

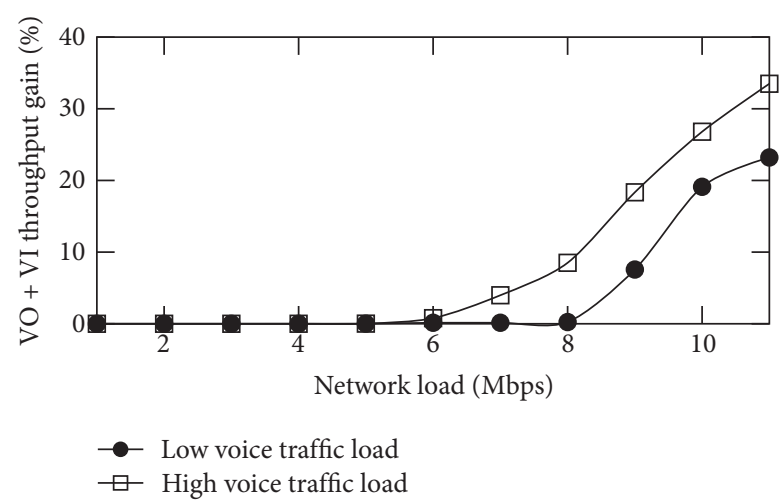

(b) Scenarios with EDCA stations

FIGURE 12: Voice + video throughput gain achieved by the dynamic scheme on the basis of two different voice traffic loads.

also presented according to the presence or absence of these legacy DCF stations in the wireless network. These results can be observed in Figures 12 and 13.

Figure 12 shows the performance gain for the two aforementioned voice traffic loads. In this figure it is clearly seen that the enhancement achieved is higher when the scenarios are only composed of stations that use EDCA, as depicted in Figure 13(b). In fact, as the network load increases, this enhancement becomes higher, being it from $30 \%$ in the case of a high voice traffic load. By contrast, in Figure 13(a) it is

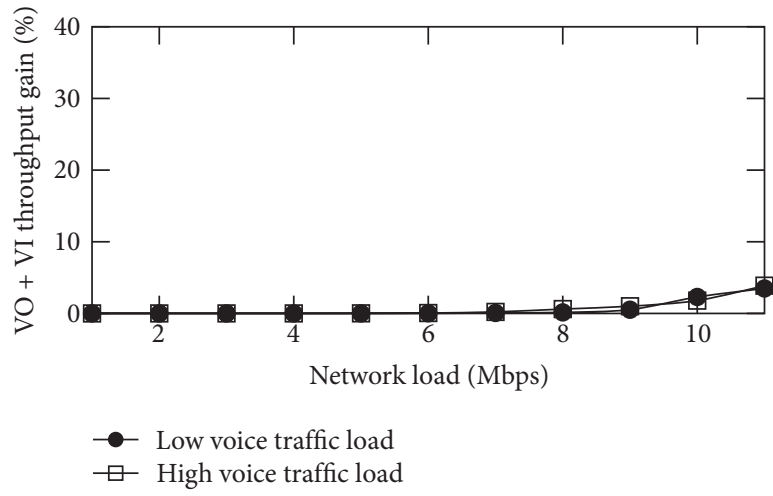

(a) Scenarios with EDCA and DCF stations

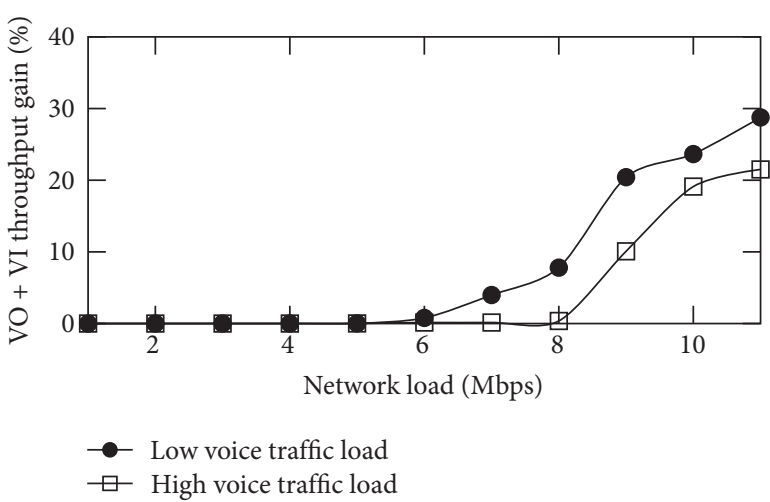

(b) Scenarios with EDCA stations

FIGURE 13: Voice + video throughput gain achieved by the dynamic scheme on the basis of two different video traffic loads.

shown that the improvement made by our scheme is slightly smaller in the presence of legacy stations. This is because, when the network holds a high DCF traffic load, the proposed scheme usually uses the default values for the parameter set, as EDCA does. Moreover, it can be also observed that further improvements are done in scenarios in which a high voice traffic load is transmitted.

Similar results are found when analyzing the behavior of the two defined video traffic loads (see Figure 13). In the case of scenarios without stations that use DCF to access 
the channel, the enhancement achieved by our proposal is markedly increased. Moreover, when a high amount of video applications are transmitted in the network, the throughput improvement is much higher. Finally, notice that, in the analysis of the video applications, the differences found between a low and a high video traffic load are lightly smaller than in the voice transmissions case.

\section{Conclusions}

In this paper, we have proposed a new dynamic prediction scheme which aims to enhance the quality of voice and video transmissions over IEEE 802.11 WLANs. This scheme dynamically adapts the AIFSN combination and the CW size to optimize the access to the medium for stations that use EDCA, while ensuring compatibility with those that only support DCF. The proposal is composed of several predictive submodels made up of J48 decision tree classifiers and M5 regression models, and it is only used by the AP of the network. As a consequence, no changes need to be made to current commercial network cards. Furthermore, periodic communication of the medium access parameters is carried out through the use of beacon frames, avoiding in this way the introduction of additional control traffic into the network.

The results have proved that the proposal improves the EDCA capacities, boosting the performance of the multimedia communications by more than $20 \%$ in some scenarios. This improvement is achieved via both suitable separation of the AIFSN values and the appropriate selection of the CW size for each AC. Furthermore, this approach leads to a reduction in the number of retransmission attempts and contributes to enhancing the global throughput of the network.

\section{Competing Interests}

The authors declare that they have no competing interests.

\section{Acknowledgments}

This work has been jointly supported by the Spanish Ministry of Economy and Competitiveness and the European Commission (FEDER funds) under Projects TIN2015-66972-C52-R and Grant BES-2013-065457.

\section{References}

[1] Wireless LAN Medium Access Control (MAC) and Physical Layer (PHY) Specifications, ANSI/IEEE Std 802.11, LAN/MAN Standards Committee of the IEEE Computer Society Std., 1999.

[2] "Wireless LAN Medium Access Control (MAC) and Physical Layer (PHY) Specifications. Amendment 7: Medium Access Control (MAC) Quality of Service (QoS)," ANSI/IEEE Std 802.11e, LAN/MAN Standards Committee of the IEEE Computer Society Std, 2005.

[3] G. Bianchi, "Performance analysis of the IEEE 802.11 distributed coordination function," IEEE Journal on Selected Areas in Communications, vol. 18, no. 3, pp. 535-547, 2000.

[4] Z. Wang and X. Guo, "Priority-based parameter performance optimization for EDCA," in Proceedings of the 3rd International
Conference on Computer Science and Network Technology (ICCSNT '13), pp. 685-688, Dalian, China, October 2013.

[5] J. Villalón, P. Cuenca, and L. Orozco-Barbosa, "On the capabilities of IEEE 802.11e for multimedia communications over heterogeneous 802.11/802.11e WLANs," Telecommunication Systems, vol. 36, no. 1, pp. 27-38, 2007.

[6] J. Hui and M. Devetsikiotis, "A unified model for the performance analysis of IEEE 802.11e EDCA," IEEE Transactions on Communications, vol. 53, no. 9, pp. 1498-1510, 2005.

[7] T. Nilsson and J. Farooq, "A novel MAC scheme for solving the QoS parameter adjustment problem in IEEE 802.11e EDCA," in Proceedings of the 9th IEEE International Symposium on Wireless, Mobile and Multimedia Networks (WoWMoM '08), pp. 1-9, Newport Beach, Calif, USA, June 2008.

[8] A. Banchs, P. Serrano, and L. Vollero, "Providing service guarantees in 802.11e EDCA WLANs with legacy stations," IEEE Transactions on Mobile Computing, vol. 9, no. 8, pp. 1057-1071, 2010.

[9] R. Achary, V. Vaityanathan, P. R. Chellaih, and N. Srinivasan, "A new QoS architecture for performance enhancement of IEEE 802.11e EDCA by contention window adaption," in Proceedings of the 4th International Conference on Computational Intelligence and Communication Networks (CICN '12), pp. 74-78, Mathura, India, November 2012.

[10] R. He and X. Fang, "A fair MAC algorithm with dynamic priority for 802.11e WLANs," in Proceedings of the International Conference on Communication Software and Networks (ICCSN '09), pp. 255-259, Macau, China, February 2009.

[11] A. Hamidian and U. Körner, "An enhancement to the IEEE 802.11e EDCA providing QoS guarantees," Telecommunication Systems, vol. 31, no. 2-3, pp. 195-212, 2006.

[12] Y.-S. Chen, M.-Y. Chuang, F.-C. Tseng, and C.-H. Ke, "High performance distributed coordination function with QoS support in IEEE 802.11e networks," in Proceedings of the Australasian Telecommunication Networks And Applications Conference (ATNAC '11), pp. 1-6, Melbourne, Australia, November 2011.

[13] P. Bellavista, A. Corradi, and L. Foschini, "Dynamic and context-aware streaming adaptation to smooth quality degradation due to IEEE 802.11 performance anomaly," The Journal of Supercomputing, vol. 45, no. 1, pp. 15-28, 2008.

[14] Z. Yuan and G.-M. Muntean, "A prioritized adaptive scheme for multimedia services over ieee 802.11 WLANs," IEEE Transactions on Network and Service Management, vol. 10, no. 4, pp. 340-355, 2013.

[15] J. R. Gallardo, S. C. Cruz, D. Makrakis, and A. Shami, "Analysis of the EDCA access mechanism for an IEEE 802.11e-compatible wireless LAN," in Proceedings of the 13th IEEE Symposium on Computers and Communications (ISCC '08), pp. 891-898, Marrakesh, Morocco, July 2008.

[16] B.-H. Lee and H.-C. Lai, "Study on bandwidth control for multiple transmission rates in IEEE 802.11e EDCAF," Wireless Communications and Mobile Computing, vol. 10, no. 8, pp. 11131127, 2010.

[17] A. Banchs and L. Vollero, "Throughput analysis and optimal configuration of 802.11e EDCA," Computer Networks, vol. 50, no. 11, pp. 1749-1768, 2006.

[18] J.-H. Wen and C.-E. Weng, "The performances study of IEEE 802.11e to support QoS in channel error environment," Wireless Communications and Mobile Computing, vol. 12, no. 15, pp. 13811388, 2012. 
[19] R. MacKenzie and T. O’Farrell, “Achieving service differentiation in IEEE 802.11e enhanced distributed channel access systems," IET Communications, vol. 6, no. 7, pp. 740-750, 2012.

[20] S. Weisberg, Applied Linear Regression, John Wiley \& Sons, Hoboken, NJ, USA, 3rd edition, 2005.

[21] S. Haykin, Neural Networks and Learning Machines, Prentice Hall, 3rd edition, 2009.

[22] B. E. Boser, I. M. Guyon, and V. N. Vapnik, "Training algorithm for optimal margin classifiers," in Proceedings of the 5th Annual ACM Workshop on Computational Learning Theory, pp. 144152, Pittsburgh, Pa, USA, July 1992.

[23] R. J. Quinlan, "Learning with continuous classes," in Proceedings of the Conference on Artificial Intelligence, pp. 343-348, San Jose, Calif, USA, 1992.

[24] J. R. Quinlan, C4.5: Programs for Machine Learning, Morgan Kaufmann, 1993.

[25] Machine Learning Group at the University of Waikato, Weka 3.7.0, 2014.

[26] E. Coronado, J. Villalon, and A. Garrido, "Dynamic AIFSN tuning for improving the QoS over IEEE 802.11 WLANs," in Proceedings of the 11th International Wireless Communications and Mobile Computing Conference (IWCMC '15), pp. 73-78, August 2015.

[27] E. Coronado, J. Villalon, L. de la Ossa, and A. Garrido, "An AIFSN prediction scheme for multimedia wireless communications," in Proceedings of the 24th International Conference on Computer Communication and Networks (ICCCN '15), pp. 1-8, Las Vegas, Nev, USA, August 2015.

[28] R. Technology, Riverbed Modeler 18.0.0, 2014.

[29] ITU-T, "Coding of speech at $16 \mathrm{Kbit} / \mathrm{s}$ Uing Low-delay Code Excited Linear Prediction," 2013.

[30] ISO/IEC and ITU-T, Advanced Video Coding for Generic Audiovisual Services, Recommendation H.264 and ISO/IEC 14496-10 (MPEG-4 AVC), 2003.

[31] R. J. Punnoose, P. V. Nikitin, and D. D. Stancil, "Efficient simulation of ricean fading within a packet simulator," in Proceedings of the IEEE International Conference on Vehicular Technology, pp. 764-767, Boston, Mass, USA, 2000.

[32] "Wireless LAN Medium Access Control (MAC) and Physical Layer (PHY) Specifications. Amendment 4: Further Higher Data Rate Extension in the $2.4 \mathrm{GHz}$ Band," ANSI/IEEE Std 802.11g, LAN/MAN Standards Committee of the IEEE Computer Society Std, 2003. 


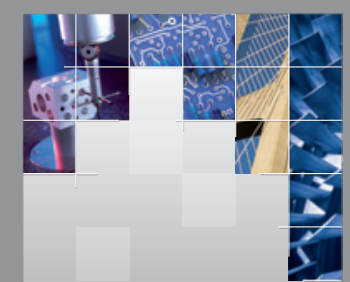

\section{Enfincering}
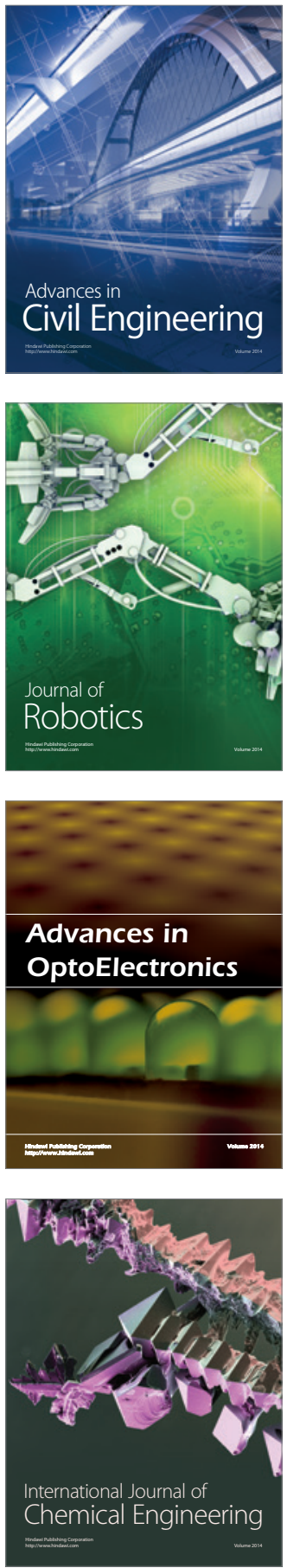

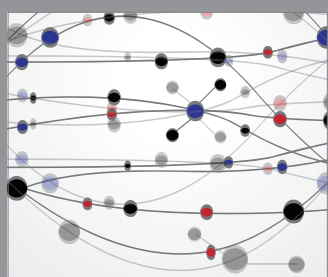

The Scientific World Journal

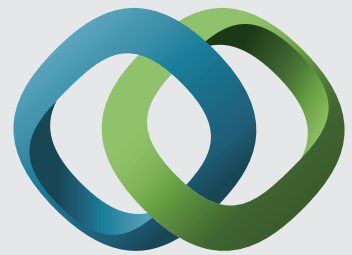

\section{Hindawi}

Submit your manuscripts at

https://www.hindawi.com
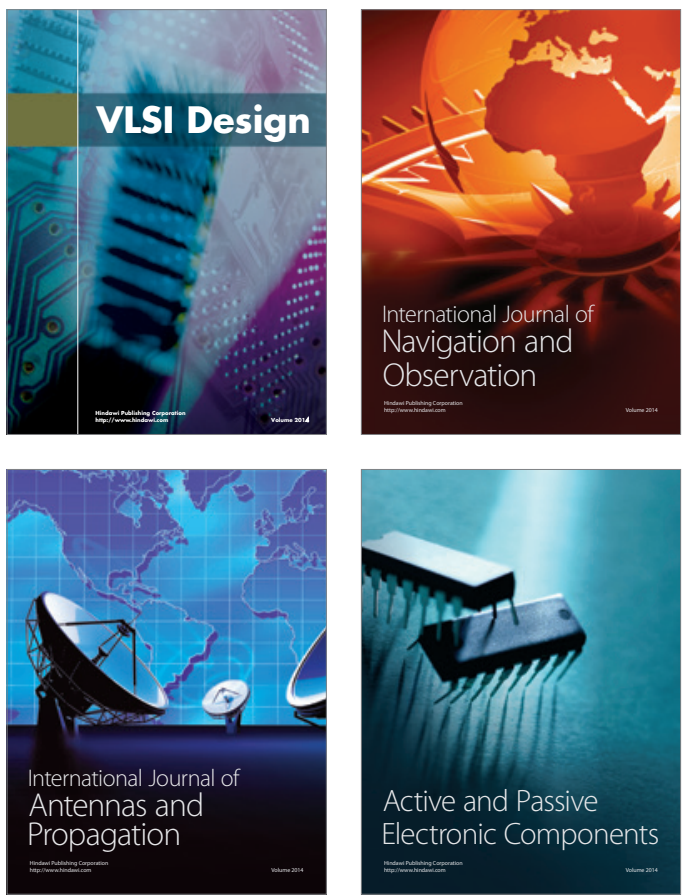
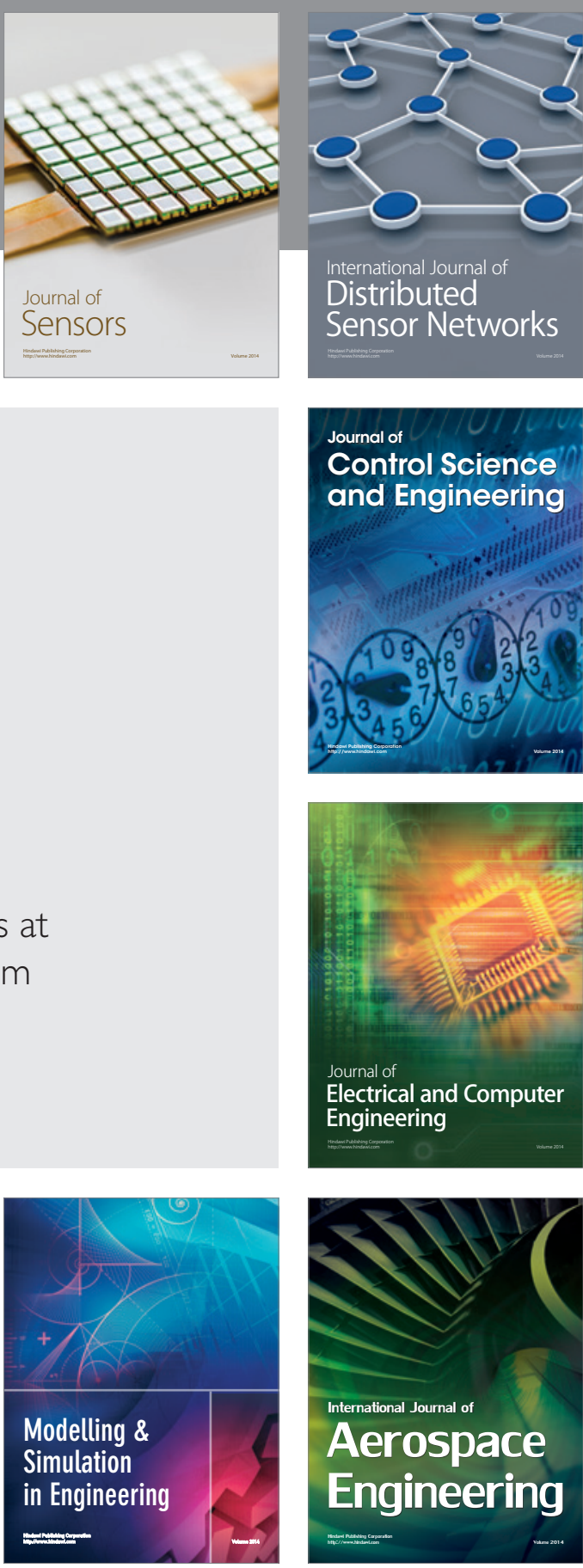

International Journal of

Distributed

Sensor Networks

$-$

Joumal of

Control Science

and Engineering
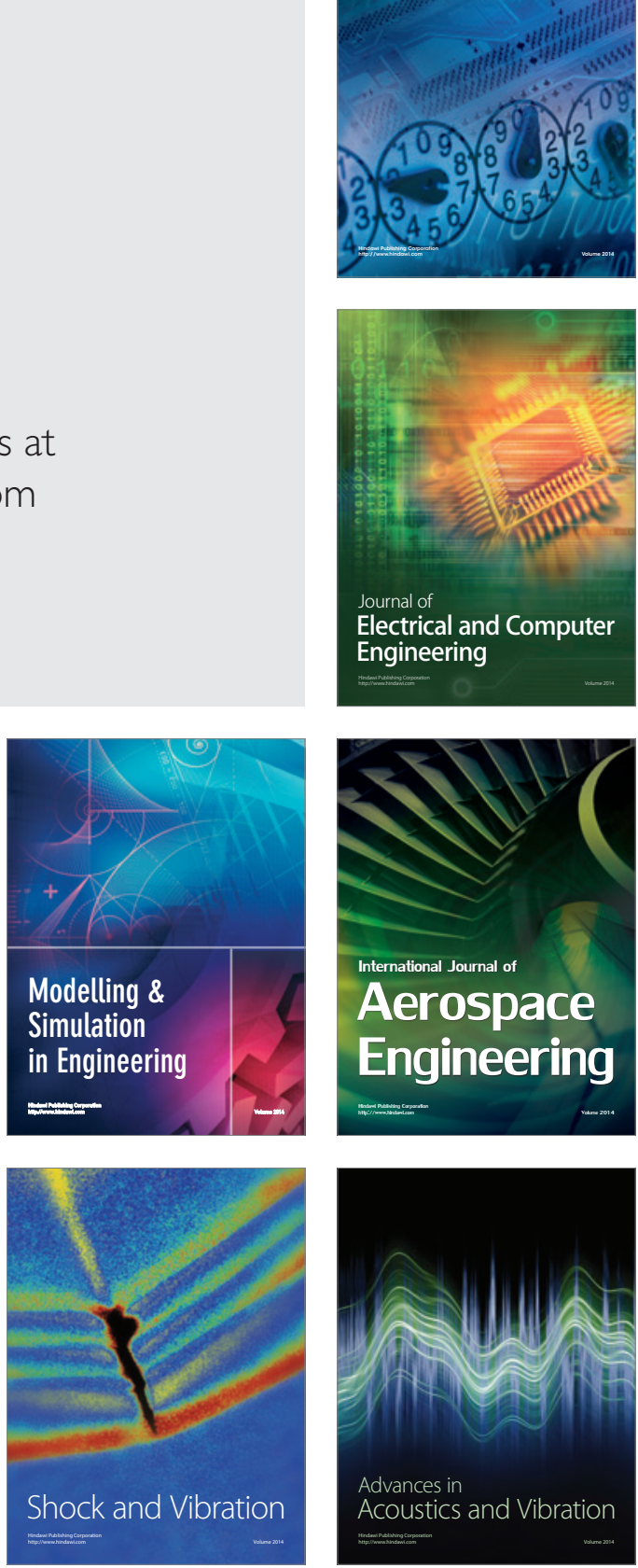